\title{
STATE ESTIMATORS FOR SOME EPIDEMIOLOGICAL SYSTEMS $*$
}

\author{
A. IGGIDR ${ }^{1}$ AND M.O. SOUZA ${ }^{2,+}$
}

\begin{abstract}
We consider a class of epidemiological models that includes most wellknown dynamics for directly transmitted diseases, and some reduced models for indirectly transmitted diseases. We then propose a simple observer that can be applied to models in this class. The error analysis of this observer leads to a non-autonomous error equation, and a new bound for fundamental matrices is also presented. We analyse and implement this observer in two examples: the classical SIR model, and a reduced Bailey-Dietz model for vector-borne diseases. In both cases we obtain arbitrary exponential convergence of the observer. For the latter model, we also applied the observer to recover the number of susceptible using dengue infection data from a district in the city of Rio de Janeiro.
\end{abstract}

\section{INTRODUCTION}

1.1. Background. From the early work by Ross and McDonald (Ross, 1911; Kermack and McKendrick, 1927; Smith et al., 2012) to more recent texts (Anderson and May, 1991; Capasso and Capasso, 1993; Diekmann and Heesterbeek, 2000; Hethcote, 2000) compartmental models have been enjoying a long and successful history in mathematical epidemiology — for instance different models have been used to assess ongoing outbreaks as in the recent Ebola epidemics (Fisman et al., 2014; Towers et al., 2014). Thus it is not surprising that this area has seen an increasing development of models - e.g. see Reiner et al. (2013) for a survey on models for vector-borne diseases. These models range from the classical ones - SI/SIS models (Susceptible-Infective); SIR (with a Removed class); or even SEIR (with an exposed class) to more complex multigroup or multipatch models (Lajmanovich and Yorke, 1976; Arino and van den Driessche, 2003; Guo et al., 2006, 2008; Iggidr et al., 2012, 2016), and models with age-structure (Pongsumpun and Tang, 2003; Inaba, 1990; Kuniya, 2011) to name only models for directly transmitted diseases. When dealing with vector-borne diseases a classical model is the SIR-SI (SIR for the hosts, and SI for the vectors) also known as the Bailey-Dietz model (Bailey, 1975; Dietz,

1Université de Lorraine, CNRS, INRIA, IECL, F-57000 Metz, France,

${ }^{2}$ Ime, Universidade Federal Fluminense, Rua Prof. Marcos Waldemar de Freitas Reis, S/N, Campus do Gragoatá, Niterói, RJ 24210-201, Brazil,

E-mail addresses: abderrahman.iggidr@inria.fr, maxsouza@id.uff.br.

Date: This version: July 16, 2018.

Key words and phrases. dengue; epidemic models; observers; state estimation.

* This work was partially funded by Inria and CAPES-Brazil in the framework of the program STIC AmSud (project MOSTICAW).

${ }^{+}$MOS was partially supported by CNPq grants \# 308113/2012-8, \# 486395/2013-8 and \# 309079/2015-2. 
1975) which, besides its simplicity, was only completely mathematically analysed 23 years later - cf. Esteva and Vargas (1998).

Besides the various modelling assumptions underlying their derivation, practical use of such models rely also on two other independent assumptions: (i) the model parameters are known (ii) an appropriate initial condition or alternatively the current state of the population is known. In both cases, knowledge of these quantities is expected to be within a given degree of certainty. Identifying these quantities from field data is an important issue, and leads to two (not necessarily independent) problems: (i) calibration of the models (or alternatively an estimation of their parameters); (ii) estimation of the current population state. Both problems are known to epidemiologists (Bjørnstad et al., 2002; Cauchemez et al., 2004; Cauchemez and Ferguson, 2008; Lloyd, 2001), but the modelling community seems to be more aware of (i) rather than (ii).

Problem (i) is certainly important, but it will not be addressed here - see for instance Toni et al. (2009); Jacquez et al. (1985); Jacquez and Greif (1985); Audoly et al. (1998). In this work, we will assume that all model parameters are known, that we have a good estimate of the total population $N(t)$, and that we have an estimate of the current number of infective individuals in the population - the $I$ compartment - with a reasonable degree of certainty. Concerning these assumptions, we notice the following: (a) the first one is a simplifying working assumption, so that we can concentrate on problem (ii); (b) rather detailed estimates of population size are usually available in many countries from their periodic census; (c) while this data is usually available through the public health authorities, its quality can be widely variable: notification delays, under or over notification, irreversible data aggregation, lack of transparency of the public health authority are some of the usual problems, only to name a few. In most situations, the available information is the number of new cases, and hence this data needs to be treated in order to yield the required estimate.

Once a model is selected, under the above assumptions, one can proceed to identify the current population state. In most classical models the total population is conserved and this yields an estimate of one of the compartments in terms of estimates of the remaining ones. Therefore, for the simplest SI/SIS models with only two compartments one immediately obtains the current population state. For the also classical SIR model, this further information only yields $S+R$. To obtain the missing estimates, there are a number of approaches (Cauchemez and Ferguson, 2008; Becker, 1989). From a more practical point of view, a possibility is to perform serological tests in the population in order to estimate the $S$ and $R$ fraction of the population - cf. Joseph et al. (1995); Drakeley et al. (2005); Gérardin et al. (2008).

1.2. State observers. An alternative approach comes from automatic and control theory: the use of so-called state observers or state estimators. In the present context, an observer can be viewed as an auxiliary dynamical system $\hat{\Sigma}$ designed to provide estimates of the complete state of another system $\Sigma$-in this case the epidemiological model of interest - using the information given by partial measurements of the state of $\Sigma$. Naturally a sine qua non condition for an observer is to converge to the true state for large time. However, in epidemiological applications such convergence should be also 
fast - ideally within a couple of weeks - see the discussion below. From a mathematical point of view, this usually means that the observer has an exponential convergence. For a more comprehensive treatment of the subject we refer to Iggidr (2004) ( a short survey of observability and observers with applications to various life-support-systems examples), Besançon (2007) (a comprehensive text that gives a general overview of observer tools for non-linear systems). See also Luenberger (1971); Gauthier et al. (1992) and the discussion in Section 2.3.

Although the literature on observer design for various dynamical systems is vast, there are fewer works on the construction of observers for epidemiological systems. Earlier work using observers in an epidemiological context dates back at least to the works of Velasco-Hernandez and collaborators (Alvarez-Ramirez et al., 2000; Velasco-Hernández et al., 2002, for an HIV model); since 2012 there is a growing interest in the literature, and more recent work includes: Bichara et al. (2014) (for a malaria's intra-host model), Tami et al. (2013); Abdelhedi et al. (2014, 2016); De la Sen et al. (2011) (for some SEIR models), Tami et al. (2014) (for a SI-SI Dengue epidemic model), Diaby et al. (2015) (for a schistosomiasis infection model), Bliman and D'Avila Barros (2017); Aronna and Bliman (2018); Bliman et al. (2018) (interval-observer for uncertain SIR and SIR-SI models) and De la Sen et al. (2011); Alonso-Quesada et al. (2012); Ibeas et al. (2015) (for SEIR models with vaccination in discrete and continuous time with vaccination)

From a practical point of view, the ideal observer ought to have two important features: (i) it should be easily implementable; (ii) it should be an efficient observer. The former feature is important if this tool is to become widely used by epidemiologists. The latter means that it converges fast, typically exponentially fast, such that one does not need a exceedingly large time series to estimate the population state. The available observers so far seems to lack either one or both of these features. Our goal in this work is to derive a simple observer that can be used for a large class of epidemiological systems, and which has exponential convergence.

1.3. Outline. In Section 2 we introduce a class of epidemiological models that includes most of systems found in the literature for directly transmitted diseases, and some reduced models for indirectly transmitted ones. For these models, we assume that the fraction of infected individuals is a measurable output of the system. We also present some examples in such class, and recall the precise definition of an observer. In Section 3, we discuss the construction of a number of observers for the generalised SIR model. Motivated by the simplest observer studied in the previous section, we introduce and analyse a specific class of observers in Section 4. This class leads to a non-autonomous error equation, which we treat using a novel bound for fundamental matrices. The theory is then applied to the observer for the generalised SIR model yielding arbitrary exponential convergence based on a tuning parameter. Section 5.2 illustrates the use of the proposed observer constructed for a reduced dengue model to have a dynamical estimate of the proportion of susceptible individuals using real data from the district of Jacarepaguá in the city of Rio de Janeiro. A discussion of the results is given in Section 6 . 


\section{EPIDEMIOLOGICAL MODELS AND OBSERVERS}

Throughout this section, $x$ will denote the population state, and $y$ the measurable information of this state. We will also write $\mathcal{M}_{m, n}(\mathbb{R})$ for the set of $m \times n$ matrices with real entries, and $\mathcal{M}_{n}(\mathbb{R})=\mathcal{M}_{n, n}(\mathbb{R})$.

The introduction of $y$ is due to the fact that usually we do not have access to the whole state: we can observe or measure only a part of the actual state of the system.

For epidemiological models, the information that is typically available is the number of new cases, and this can be usually converted in an estimate about the number of infected individuals in the population.

2.1. A class of epidemiological models. We consider population models of the form

$$
\left\{\begin{array}{l}
\dot{x}=A x+b+G(y) x \\
y=C x
\end{array}\right.
$$

where $x \in \mathbb{R}^{n}$ is the population state and $y \in \mathbb{R}^{m}$, with $m \leq n$, is the measurable output of System (1). In addition, $A \in \mathcal{M}_{n}(\mathbb{R}), b \in \mathbb{R}^{n}$, and $C \in \mathcal{M}_{m, n}(\mathbb{R})$ are constant, while $G: \mathbb{R}^{m} \rightarrow \mathcal{M}_{n}(R)$ is smooth.

2.2. Examples. The class described by System (1) includes most epidemiological models for directly transmitted diseases with or without a latent or exposed class. In particular, it includes the classical epidemiological models as the $\mathrm{S}^{*}$ and $\mathrm{S}^{*} \mathrm{~S}$ models, where ${ }^{*}$ can be I, EI, IR or EIR - together with their multigroup counterparts. In these models we will assume that the measured output is the infected class.

As an example, let us consider the multigroup SIR and SEIR models studied in Guo et al. (2006) and Guo et al. (2008), respectively. The latter is given by

$$
\left\{\begin{aligned}
\dot{S}_{k} & =\Lambda_{k}-d_{k}^{S} S_{k}-\sum_{j=1}^{n} \beta_{k j} S_{k} I_{j} \\
\dot{E}_{k} & =\sum_{j=1}^{n} \beta_{k j} S_{k} I_{j}-\left(d_{k}^{E}+\gamma_{k}\right) E_{k} \\
\dot{I}_{k} & =\gamma_{k} E_{k}-\left(d_{k}^{I}+r_{k}\right) I_{k} \\
\dot{R}_{k} & =r_{k} I_{k}-d_{k}^{R} R_{k}
\end{aligned}\right.
$$

If we write $y=I=\left(I_{1}, \ldots, I_{n}\right)^{T} \in \mathbb{R}^{n}$, and $x \in \mathbb{R}^{4 n}$. In this case we can take

$$
A=\left(\begin{array}{cccc}
-\operatorname{diag}\left(d^{S}\right) & 0 & 0 & 0 \\
0 & -\operatorname{diag}\left(d^{E}+\gamma\right) & 0 & 0 \\
0 & 0 & -\operatorname{diag}\left(d^{I}+r\right) & 0 \\
0 & 0 & 0 & -\operatorname{diag}\left(d^{R}\right)
\end{array}\right)
$$




$$
b^{t}=\left(\Lambda_{1}, \ldots, \Lambda_{n}, 0, \ldots, 0\right), \quad G(y)=\left(\begin{array}{cccc}
\widetilde{G}(y) & 0 & 0 & 0 \\
-\widetilde{G}(y) & 0 & 0 & 0 \\
0 & 0 & 0 & 0 \\
0 & 0 & 0 & 0
\end{array}\right),
$$

and $\widetilde{G}(y)$ is a diagonal matrix with

$$
G_{k k}=\sum_{j=1}^{n} \beta_{k j} y_{j}, \quad k=1, \ldots, n .
$$

Finally, we have

$$
C=\left(\begin{array}{llll}
0 & I_{n} & 0 & 0
\end{array}\right), \quad \text { where } I_{n} \text { is the } n \times n \text { identity matrix. }
$$

This class also includes some reduced models for indirectly transmitted diseases. As an example, we can mention the fast-vector regime of the Bailey-Dietz model discussed in Souza (2014) — but see also the discussion in Keeling and Rohani (2008) — given by

$$
\left\{\begin{array}{l}
\dot{x}_{1}=\mu_{h}\left(1-x_{1}\right)-f\left(x_{2}\right) x_{1} \\
\dot{x}_{2}=-\left(\gamma+\mu_{h}\right) x_{2}+f\left(x_{2}\right) x_{1}
\end{array}=\left(\begin{array}{c}
F_{1}(x) \\
F_{2}(x)
\end{array}\right),\right.
$$

with $f(s)=\frac{\delta \sigma s}{\mu_{v}+\sigma s}$. Here, $x_{1}$ is the fraction of susceptible in the host population, while $x_{2}=y$ is the fraction of infectious, assumed measurable, in the same population.

For System (2):

$A=\left(\begin{array}{cc}-\mu_{h} & 0 \\ 0 & -\left(\gamma+\mu_{h}\right)\end{array}\right), G(y)=\left(\begin{array}{cc}-f(y) & 0 \\ f(y) & 0\end{array}\right), b^{T}=\left(\mu_{h}, 0\right)$, and $C=\left(\begin{array}{ll}0 & 1\end{array}\right)$.

2.3. Epidemiological observers. An observer for (1) is a dynamical system

$$
\left\{\begin{array}{l}
\dot{z}(t)=\hat{F}(z(t), y(t)) \\
\hat{x}(t)=L(z(t), y(t))
\end{array}\right.
$$

whose solutions satisfy $|\hat{x}(t)-x(t)|$ goes to zero as $t \rightarrow \infty$ for any initial conditions $x_{0}$ and $z_{0}$. When the convergence of $\hat{x}(t)$ towards $x(t)$ is exponential, the system (3) is an "exponential observer". More precisely, system (3) is an exponential observer for system (1) if there exists $\lambda>0$ such that, for all $t \geq 0$ and for all initial conditions $(x(0), z(0))$, the corresponding solutions of (1)-(3) satisfy

$$
|\hat{x}(t)-x(t)| \leq e^{-\lambda t}|\hat{x}(0)-x(0)| .
$$

In order to obtain the observer (3), one needs to specify the vector field $\hat{F}$ and the map $L$.

We must point out that the initial state $x_{0}$ of (1) is unknown while the initial state $z_{0}$ of the observer can be assigned arbitrarily. 


\section{The SIR MOdel AS An EXAmple}

3.1. The SIR model with generalised incidence. The standard SIR (SusceptibleInfectious-Removed) model considers a homogeneous population which is divided into three classes $S, I$ and $R$ according to the health state of its individuals. Let $S(t), I(t)$ and $R(t)$ denote the number of individuals in the corresponding class at time $t$.

The disease dynamics is given by

$$
\left\{\begin{aligned}
\dot{S} & =\mu(N-S)-g(I) S \\
\dot{I} & =g(I) S-(\mu+\gamma) I \\
\dot{R} & =\gamma I-\mu R
\end{aligned}\right.
$$

with $g$ assumed to be at least $C^{1}$, with $g(0)=0$ and $g^{\prime}(0)>0$ - hence, by Hadamard Lemma, we can write $g(I)=I \hat{g}(I)$, with $\hat{g}(0)>0$.

For simplicity, we will assume that the system is at its carrying capacity, i.e. $S+I+$ $R=N$, and hence if we let $x_{1}=S / N, x_{2}=I / N$, and abuse language by writing $g\left(x_{2}\right)=g\left(N x_{2}\right)$, we arrive at

$$
\left\{\begin{array}{l}
\dot{x}_{1}=\mu\left(1-x_{1}\right)-g\left(x_{2}\right) x_{1} \\
\dot{x}_{2}=g\left(x_{2}\right) x_{1}-(\mu+\gamma) x_{2}
\end{array}\right.
$$

The choice $g\left(x_{2}\right)=\beta x_{2}$ in System (5) leads to the standard SIR model, while choosing $g\left(x_{2}\right)=\frac{\sigma \delta x_{2}}{\mu_{v}+\sigma x_{2}}$ recovers the reduced Bailey-Dietz model (2).

In particular, System (5) belongs to the class of systems described by (1), if we assume that the measurable output is $y=x_{2}$. The question we pose now is how to use the measurable output $y(t)$ together with System (5) in order to obtain an estimate of the value of $x_{1}(t)$. In what follows, we will now describe a number of possible observers for this system that might accomplish this task.

3.2. High-gain observer. A well known construction method in non-linear systems is the high-gain method, which is based on the use of a suitable non-linear coordinate change.

The phase space for System (5) is given by:

$$
\Omega=\left\{0 \leq x_{1}, x_{2} \leq 1, x_{1}+x_{2} \leq 1\right\} .
$$

Let

$$
\phi\left(x_{1}, x_{2}\right)=\left(\begin{array}{c}
x_{2} \\
x_{1} g\left(x_{2}\right)-\left(\mu_{h}+\gamma\right) x_{2}
\end{array}\right) .
$$

The Jacobian of $\phi$ is:

$$
D \phi=\left(\begin{array}{cc}
0 & 1 \\
g\left(x_{2}\right) & g^{\prime}\left(x_{2}\right) x_{1}-\mu_{h}-\gamma
\end{array}\right) .
$$


Let us introduce the following coordinates change:

$$
\left\{\begin{array}{l}
w=\phi\left(x_{1}, x_{2}\right) \text { i.e. } \\
w_{1}=y=x_{2} \\
w_{2}=\dot{y}=x_{1} g\left(x_{2}\right)-\left(\mu_{h}+\gamma\right) x_{2} \\
x_{1}=\frac{\mu_{h} w_{1}+\gamma w_{1}+w_{2}}{g\left(w_{1}\right)} \\
x_{2}=w_{1} .
\end{array}\right.
$$

Let $\epsilon>0$ and $\Omega_{\epsilon}=\left\{0<x_{1}<1, \epsilon<x_{2}<1, x_{1}+x_{2} \leq 1\right\}$. $\phi$ is a diffeomorphism from $\Omega_{\epsilon}$ to $\phi\left(\Omega_{\epsilon}\right)$. It can be proved that there exists $\epsilon>0$ such that $\Omega_{\epsilon}$ is positively invariant. System (2) is globally Lipschitz on $\Omega_{\epsilon}$.

With the new coordinates, system (2) is given by:

$$
\left\{\begin{array}{l}
\dot{w}_{1}=w_{2}, \\
\dot{w}_{2}=\psi(w), \\
y=w_{1},
\end{array}\right.
$$

with

$$
\psi(w)=\frac{\mu g\left(w_{1}\right)+\left[(\mu+\gamma) w_{1}+w_{2}\right]\left[g^{\prime}\left(w_{1}\right) w_{2}-\mu g\left(w_{1}\right)\right]}{g\left(w_{1}\right)}-g^{2}\left(w_{1}\right)-(\mu+\gamma) w_{2} .
$$

For the particular case of the reduced Bailey-System model given by Equation (2), we have (replacing $\mu$ by $\mu_{h}$ ) that:

$$
\begin{aligned}
\psi(w) & =\frac{-\left(\gamma \mu_{h} \mu_{v}+w_{1} \gamma\left(\delta+\mu_{h}\right) \sigma+\mu_{h}\left(\left(w_{1}-1\right) \delta \sigma+\mu_{h}\left(\mu_{v}+w_{1} \sigma\right)\right)\right) w_{1}^{2}}{w_{1}\left(\mu_{v}+w_{1} \sigma\right)} \\
& +\frac{-w_{2}\left(\mu_{h} \mu_{v}+w_{1}\left(\gamma+\delta+2 \mu_{h}\right) \sigma\right) w_{1}+w_{2}^{2} \mu_{v}}{w_{1}\left(\mu_{v}+w_{1} \sigma\right)} \\
& =\delta \mu_{h}-\left(\gamma \delta+\gamma \mu_{h}+\delta \mu_{h}+\mu_{h}^{2}\right) w_{1}-\left(\gamma+\delta+2 \mu_{h}\right) w_{2} \\
& +\frac{\mu_{v} w_{2}^{2}+\left(-\delta \mu_{h} \mu_{v}+\left(\gamma \mu_{v}+\delta \mu_{v}+\mu_{h} \mu_{v}\right) w_{2}\right) w_{1}+\left(\gamma \delta \mu_{v}+\delta \mu_{h} \mu_{v}\right) w_{1}^{2}}{w_{1}\left(\mu_{v}+w_{1} \sigma\right)} .
\end{aligned}
$$

It is, at least theoretically, possible to use a high-gain observer since system (6) has the "good" form. The high-gain observer is given by (see, for instance, Gauthier et al., 1992):

$$
\left\{\begin{array}{l}
\dot{\hat{w}}_{1}=\hat{w}_{2}-2 \theta\left(\hat{w}_{1}-y\right), \\
\dot{\hat{w}}_{2}=\psi(\hat{w})-\theta^{2}\left(\hat{w}_{1}-y\right), \\
\hat{x}(t)=\phi^{-1}(w(t)),
\end{array}\right.
$$


where $\theta$ is the tuning gain of the observer. It has been proved in (Gauthier et al., 1992) that system (7) is an exponential observer for system (6) if $\psi$ is globally Lipschitz on $\mathbb{R}^{2}$ and $\theta$ large enough. Moreover the error convergence rate can be chosen arbitrary fast by increasing the value of $\theta$.

The function $\psi$ is globally Lipschitz on $\phi\left(\Omega_{\epsilon}\right)$. The domain $\phi\left(\Omega_{\epsilon}\right)$ is positively invariant for system (6) but it is not positively invariant for the candidate observer (7).

Therefore, one needs to extend $F($ or $\psi$ ) as well as the diffeomorphism $\phi$ to the whole $\mathbb{R}^{2}$ such that the respective prolongations are globally Lipschitz. It is known that these prolongations do exist but the problem is that the observer construction involves their explicit form. It is not a difficult problem to find the prolongation of the vector field $F$. However, it is rather a hard task to construct explicitly a globally Lipschitz prolongation for the diffeomorphism $\phi$.

In the original coordinates, the high-gain observer (7) is given by:

$$
\left\{\begin{array}{l}
\dot{z}_{1}=F_{1}(z)-\frac{\theta\left(\mu_{v}+\sigma z_{2}\right)}{\delta \sigma z_{2}}\left(\theta+2\left(\gamma+\mu_{h}-\frac{\delta \mu_{v} \sigma z_{1}}{\left(\mu_{v}+\sigma z_{2}\right)^{2}}\right)\right)\left(z_{2}-y\right), \\
\dot{z}_{2}=F_{2}(z)-2 \theta\left(z_{2}-y\right) \\
\hat{x}(t)=z(t) .
\end{array}\right.
$$

Unlike system (2) for which one can ensure that $x_{2}(t)>\epsilon$, nothing guarantee that $z_{2}(t)>\epsilon$. Hence, system (8) could be not well defined. The same remark can be done for the high-gain observer $(7): \hat{w}_{1}(t)$ can vanish and hence $\psi(\hat{w}(t))$ is not defined nor $\phi^{-1}(\hat{w}(t))$. That's why it is necessary to extend the vector field as well as the diffeomorphism to the whole $\mathbb{R}^{2}$ before constructing the high-gain observer for our model. An example showing that the high-gain observer may fail to converge, when the Lipschitz extension is not used, is given in Guiro et al. (2009).

3.3. A first alternative observer. System (5) can be written

$$
\left\{\begin{array}{l}
\dot{x}_{1}=\mu_{h}\left(1-x_{1}\right)-g(y) x_{1} \\
\dot{x}_{2}=-\left(\gamma+\mu_{h}\right) x_{2}+g(y) x_{1} \\
y=x_{2}
\end{array}\right.
$$

The first proposed "simple" observer is:

$$
\left\{\begin{array}{l}
\dot{z}_{1}=\mu_{h}\left(1-z_{1}\right)-g(y) z_{1}-\frac{1}{k_{1}+g(y)}\left(z_{2}-y\right), \\
\dot{z}_{2}=-\left(\gamma+\mu_{h}\right) z_{2}+g(y) z_{1}-k_{2}\left(z_{2}-y\right) .
\end{array}\right.
$$

The observer tuning parameters are $k_{1}$ and $k_{2}$ and will be chosen later to ensure the convergence. 
The estimation error is $e=z-x$. It satisfies the following equation:

$$
\left\{\begin{array}{l}
\dot{e}_{1}=-\mu_{h} e_{1}-g(y) e_{1}-\frac{1}{k_{1}+g(y)} e_{2} \\
\dot{e}_{2}=-\left(\gamma+\mu_{h}\right) e_{2}+g(y) e_{1}-k_{2} e_{2}
\end{array}\right.
$$

We take as a candidate Lyapunov function:

$$
V=\frac{1}{2}\left(e_{1}^{2}+e_{2}^{2}\right) .
$$

Its time derivative is:

$$
\begin{aligned}
\dot{V} & =-\left(\mu_{h}+g(y)\right) e_{1}^{2}+\left(g(y)-\frac{1}{k_{1}+g(y)}\right) e_{1} e_{2}-\left(\gamma+\mu_{h}+k_{2}\right) e_{2}^{2} \\
& =-\left(\mu_{h}+g(y)\right)\left(e_{1}-\frac{g(y)-\frac{1}{k_{1}+g(y)}}{2\left(\mu_{h}+g(y)\right)} e_{2}\right)^{2}-\left(\gamma+\mu_{h}+k_{2}-\frac{\left(g(y)-\frac{1}{k_{1}+g(y)}\right)^{2}}{4\left(\mu_{h}+g(y)\right)} e_{2}^{2} .\right.
\end{aligned}
$$

Thus $\dot{V}<0$ if $k_{2}$ can be chosen in such a way that:

$$
\gamma+\mu_{h}+k_{2}-\frac{\left(g(y)-\frac{1}{k_{1}+g(y)}\right)^{2}}{4\left(\mu_{h}+g(y)\right)}>0 \text { for all } y \in(0,1) .
$$

Notice that under our assumptions, $g$ is positive and bounded in $(0,1)$.

Indeed, assume that $k_{1}>0$, and let $M\left(k_{1}\right)$ be the maximum value of $\frac{\left(g(y)-\frac{1}{k_{1}+g(y)}\right)^{2}}{4\left(\mu_{h}+g(y)\right)}$ in $[0,1]$. Then choose $k_{2}$ such that $k_{2}>M\left(k_{1}\right)-\left(\gamma+\mu_{h}\right)$.

Then, we have $\dot{V}<0$. Hence, since $V \geq 0$, we have that there exists $L \geq 0$ such that

$$
\lim _{t \rightarrow \infty} V\left(e_{1}(t), e_{2}(t)\right)=L .
$$

In addition, we have also that

$$
\lim _{t \rightarrow \infty} \dot{V}(t)=0
$$

From the computed functional form of $\dot{V}$, we then have that

$$
\begin{array}{r}
\lim _{t \rightarrow \infty}\left(\mu_{h}+g(y)\right)\left(e_{1}-\frac{g(y)-\frac{1}{k_{1}+g(y)}}{2\left(\mu_{h}+\gamma\right)} e_{2}\right)^{2}=0, \\
\lim _{t \rightarrow \infty}\left(\gamma+\mu_{h}+k_{2}-\frac{\left(g(y)-\frac{1}{k_{1}+g(y)}\right)^{2}}{4\left(\mu_{h}+g(y)\right)}\right)_{2}^{2}=0,
\end{array}
$$


bearing in mind that $y, e_{1}$ and $e_{2}$ are functions of $t$. Hence, from the second limit above, we have that

$$
\lim _{t \rightarrow \infty} e_{2}(t)=0,
$$

and from this, and the first equation above, we also have that

$$
\lim _{t \rightarrow \infty} e_{1}(t)=0 .
$$

However, it does not follow immediately that the convergence of this observer will be exponential. We thus turn our attention to an even simpler observer.

\subsection{A second observer. Simply:}

$$
\left\{\begin{array}{l}
\dot{z}_{1}=\mu_{h}\left(1-z_{1}\right)-g(y) z_{1}-k_{1}\left(z_{2}-y\right), \\
\dot{z}_{2}=-\left(\gamma+\mu_{h}\right) z_{2}+g(y) z_{1}-k_{2}\left(z_{2}-y\right) .
\end{array}\right.
$$

The observer tuning parameters are $k_{1}$ and $k_{2}$ and will be chosen later to ensure the convergence. The estimation error is $e=z-x$. It satisfies the following equation:

$$
\left\{\begin{array}{l}
\dot{e}_{1}=-\mu_{h} e_{1}-g(y) e_{1}-k_{1} e_{2}, \\
\dot{e}_{2}=-\left(\gamma+\mu_{h}\right) e_{2}+g(y) e_{1}-k_{2} e_{2} .
\end{array}\right.
$$

We take as a candidate Lyapunov function:

$$
V=\frac{1}{2}\left(e_{1}^{2}+e_{2}^{2}\right) .
$$

Its time derivative is:

$$
\begin{aligned}
\dot{V} & =-\left(\mu_{h}+f(y)\right) e_{1}^{2}+\left(g(y)-k_{1}\right) e_{1} e_{2}-\left(\gamma+\mu_{h}+k_{2}\right) e_{2}^{2} \\
& =-\left(\mu_{h}+g(y)\right)\left(e_{1}-\frac{\left(g(y)-k_{1}\right)}{2\left(\mu_{h}+g(y)\right)} e_{2}\right)^{2}-\left(\gamma+\mu_{h}+k_{2}-\frac{\left(g(y)-k_{1}\right)^{2}}{4\left(\mu_{h}+g(y)\right)}\right) e_{2}^{2} .
\end{aligned}
$$

A similar argument shows that the error converges to zero, using a suitable choice of $k_{1}$ and $k_{2}$. However, once again, since both the error equation and $\dot{V}$ are non-autonomous it is not an easy task to obtain uniform exponential convergence.

\section{OBSERVERS FOR EPIDEMIOLOGICAL SYSTEMS}

4.1. A simple and effective observer class. Motivated by the second alternative observer, we introduce a candidate observer (or state estimator) for models in the class described by (1) as follows:

$$
\begin{cases}\dot{z} & =A z+b+G(y) z-K(y)(C z-y), \\ \hat{x}(t) & =z(t),\end{cases}
$$

and the corresponding error equation is given by

$$
\dot{e}=(A+G(y)-K(y) C) e=M(y) e .
$$


In order to discuss the performance of the candidate observer (14), we need to recall some definitions and classical results from Control Theory.

Definition 1 (Kalman observability rank condition (Wonham, 1979)). Let $\tilde{A}$ be a $n \times$ $n$ matrix and $\tilde{C}$ be a $q \times n$ matrix. The pair $(\tilde{A}, \tilde{C})$ is said to satisfy the Kalman observability rank condition if the matrix:

$$
\mathbf{O}_{(\tilde{C}, \tilde{A})}=\left(\begin{array}{c}
\tilde{C} \\
\tilde{C} \tilde{A} \\
\tilde{C} \tilde{A}^{2} \\
\vdots \\
\tilde{C} \tilde{A}^{n-1}
\end{array}\right)
$$

is of rank $n$.

Theorem 1 (Pole-Shifting Theorem (Wonham, 1979, page 61)). If the pair $(\tilde{A}, \tilde{C})$ satisfies the Kalman observability rank condition then for any given set $\mathcal{S}=\left\{\alpha_{1}, \ldots \alpha_{n}\right\}$ of $n$ complex numbers satisfying $z \in \mathcal{S} \Rightarrow \bar{z} \in \mathcal{S}$, it is possible to find a matrix $K$ in such a way that the spectrum of $\tilde{A}-K \tilde{C}$ is $\sigma(\tilde{A}-K \tilde{C})=\mathcal{S}$. In particular it is possible to find a matrix $K$ in such a way that the eigenvalues of the matrix $\tilde{A}-K \tilde{C}$ are all with negative real part.

If the pair $(\tilde{A}, \tilde{C})$ satisfies the Kalman observability rank condition then the associated autonomous system

$$
\left\{\begin{array}{l}
\dot{x}=\tilde{A} x, \\
y=\tilde{C} x
\end{array}\right.
$$

is observable and an exponential observer is simply given by the Luenberger observer (Luenberger, 1971) $\dot{z}=\tilde{A} z-K(\tilde{C} z-y)$ since the error $e=z-x$ is governed by $\dot{e}=(\tilde{A}-K \tilde{C}) e$ for which the zero-solution can be made globally exponentially stable by choosing the matrix $K$ in such a way that the eigenvalues of the matrix $\tilde{A}-K \tilde{C}$ are all with negative real part.

Definition 2. We will say that System (1) is parametrically linearly observable if for any $y>0$, the pair $(A+G(y), C)$ satisfies the Kalman observability rank condition - this means that the linear system obtained by fixing y as a parameter is observable.

Under the extra condition that System (1) satisfies Definition 2, we will provide a method that can be used to establish, in many cases, the exponential convergence of the observer given by System (14).

The observer (14) is quite simple to construct (it is a Luenberger like observer). Moreover it does not involve any non-linear coordinates change.

For fixed $y$, it is well-known that the Kalman condition as given in Definition 1 is sufficient to show that the origin is a globally asymptotically stable equilibrium for Equation (15). In this case, Theorem 1 combined with Lyapunov's Theorem allows one 
to conclude that there exists a symmetric positive definite $P(y)$ such that $L=\langle P(y) e, e\rangle$ is a Lyapunov function for System (15) (with $y$ fixed). Indeed, $P(y)$ can be chosen such that

$$
\begin{aligned}
\dot{L} & =\langle P(y) M(y) e, e\rangle+\langle P(y) e, M(y) e\rangle \\
& =\left\langle\left(P(y) M(y)+M(y)^{T} P(y) e, e\right\rangle\right. \\
& =-\langle e, e\rangle .
\end{aligned}
$$

However, since $y$ does depend on $t$, this argument is not sufficient to show that $L$ is indeed a Lyapunov function. Nevertheless, a slight adaptation of the classical results proves the following: Let $P$ be a symmetric positive-definite matrix and let us define

$$
S(y)=P M(y)+M(y)^{T} P .
$$

If, for an appropriate choice of gain $K(y)$, we have that $S(y)$ is negative defined for all $y$ in some suitable domain, then $L=\langle P e, e\rangle$ is a Lyapunov function for System (15). Inasmuch as the existence of such a $y$-independent $P$ is not guaranteed, we shall use the "machinery" developed below to prove exponential convergence of the estimation error (15).

For exponential convergence, notice that if $\Phi\left(t ; t_{0}\right)$ is a fundamental matrix of $(15)$, then we need a bound of the type

$$
\left\|\Phi\left(t ; t_{0}\right)\right\| \leq \mathfrak{C}(t) \mathrm{e}^{\alpha\left(t-t_{0}\right)},
$$

where $\|\cdot\|$ is some matrix norm, $\mathfrak{C}$ is a function that grows at most polynomially, and $\alpha<0$.

4.2. A bound for fundamental matrices. We will denote the set of real $n \times n$ matrices by $\mathcal{M}_{n}$. Let $M: I \rightarrow \mathcal{M}_{n}$ be a matrix function that is Holder continuous of degree $r$, and let $\mathcal{H}$ be its Holder constant. We will denote by $\sigma(M)$ the spectrum of $\mathrm{M}$ (i.e., the set of all eigenvalues of $M$ ) and by $\alpha(M)$ the spectral abscissa (also known as the stability modulus) of the matrix $M$, i.e., the maximum among the real part of the eigenvalues of $M-$ cf. Smith (1995, page 60). In symbols, $\alpha(M)=\max \{\operatorname{Re}(\lambda), \lambda \in \sigma(M)\}$.

Let $\Phi(t)$ denote the fundamental matrix associated to $M(t)$. Then, we have

Theorem 2. Assume that the spectral abscissa of $M(\tau)$ is bounded by a constant $\alpha$-i.e that $\alpha(M(\tau)) \leq \alpha, \tau \in \mathbb{R}$. Let $\kappa(t)$ be an appropriate non-decreasing function, with at most polynomial growth, and with $\kappa(0)=1$, such that

$$
\left\|\mathrm{e}^{t M(\tau)}\right\|_{2} \leq \kappa(t) \mathrm{e}^{\alpha t} .
$$

Let

$$
\xi_{0}=\lim _{t \rightarrow 0^{+}} \frac{\log \kappa(t)}{t} \geq 0 .
$$

Then, we have two possibilities:

$\xi_{0}=0$ : in this case, for any $\eta>0$, there exists $\delta>0$ such that

$$
\|\Phi(t)\|_{2} \leq \kappa(\delta) \exp ((\alpha+\eta) t) ;
$$


$\xi_{0}>0$ : then, there exists $\bar{\delta}>0$, such that

$$
\|\Phi(t)\|_{2} \leq \kappa(\delta) \exp ((\alpha+\rho) t),
$$

with

$$
\rho=\frac{\log \kappa(\bar{\delta})}{\bar{\delta}}+\mathcal{H} \kappa(\bar{\delta}) \frac{\bar{\delta}^{r}}{2^{r}(r+1)},
$$

and the bound is optimal.

Proof. We first observe that by writing $M(t)=M(s)+M(t)-M(s)$, for some fixed $s$, we have that

$$
\Phi(t)=\mathrm{e}^{t M(s)} \Phi(0)+\int_{0}^{t} \mathrm{e}^{(t-\tau) M(s)}(M(\tau)-M(s)) \Phi(\tau) \mathrm{d} \tau .
$$

Hence

$$
\begin{aligned}
\|\Phi(t)\|_{2} & \leq \kappa(t) \mathrm{e}^{\alpha t}\|\Phi(0)\|_{2}+\int_{0}^{t}\left\|\mathrm{e}^{(t-\tau) M(s)}\right\|_{2}\|M(\tau)-M(s)\|_{2}\|\Phi(\tau)\|_{2} \mathrm{~d} \tau \\
& \leq \kappa(t) \mathrm{e}^{\alpha t}\|\Phi(0)\|_{2}+\int_{0}^{t} \kappa(t-\tau) \mathrm{e}^{\alpha(t-\tau)}\|M(\tau)-M(s)\|_{2}\|\Phi(\tau)\|_{2} \mathrm{~d} \tau .
\end{aligned}
$$

Since $\kappa$ is non-decreasing we have that i.e.

$$
\|\Phi(t)\|_{2} \leq \kappa(t) \mathrm{e}^{\alpha t}\|\Phi(0)\|_{2}+\int_{0}^{t} \kappa(t) \mathrm{e}^{\alpha(t-\tau)}\|M(\tau)-M(s)\|_{2}\|\Phi(\tau)\|_{2} \mathrm{~d} \tau,
$$

$$
\mathrm{e}^{-\alpha t}\|\Phi(t)\|_{2} \leq \kappa(t)\|\Phi(0)\|_{2}+\kappa(t) \int_{0}^{t}\|M(\tau)-M(s)\|_{2} \mathrm{e}^{-\alpha \tau}\|\Phi(\tau)\|_{2} \mathrm{~d} \tau .
$$

By Gronwall's inequality

$$
\mathrm{e}^{-\alpha t}\|\Phi(t)\|_{2} \leq \kappa(t)\|\Phi(0)\|_{2} \mathrm{e}^{\kappa(t) \int_{0}^{t}\|M(\tau)-M(s)\|_{2} \mathrm{~d} \tau},
$$

and hence it follows that

$$
\|\Phi(t)\|_{2} \leq \mathrm{e}^{\alpha t} \kappa(t)\|\Phi(0)\|_{2} \mathrm{e}^{\kappa(t) \int_{0}^{t}\|M(\tau)-M(s)\|_{2} \mathrm{~d} \tau} .
$$

By using the Holder condition of $M(t)$, and choosing $s=t / 2$, we find

$$
\|\Phi(t)\|_{2} \leq \mathrm{e}^{\alpha t} \kappa(t)\|\Phi(0)\|_{2} \mathrm{e}^{\mathcal{H} \kappa(t) \int_{0}^{t}|\tau-t / 2|^{r} \mathrm{~d} \tau} .
$$

and on noting that $\int_{0}^{t}|\tau-t / 2|^{r} \mathrm{~d} \tau=\frac{t^{r+1}}{2^{r}(r+1)}$, we may rewrite it as

$$
\|\Phi(t)\|_{2} \leq \kappa(t) \mathrm{e}^{\alpha t} \mathrm{e}^{\mathcal{H} \kappa(t) \frac{t^{r+1}}{2^{r}(r+1)}}\|\Phi(0)\|_{2} .
$$

For $0 \leq t \leq \delta$, we have

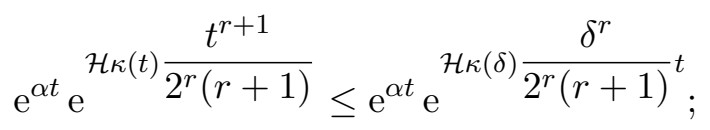


hence, we get

$$
\|\Phi(t)\|_{2} \leq \kappa(\delta) \mathrm{e}^{\alpha t} \mathrm{e}^{\mathcal{H} \kappa(\delta) \frac{\delta^{r}}{2^{r}(r+1)} t} \cdot\|\Phi(0)\|_{2}
$$

Let

$$
g_{1}(\delta)=\frac{\log \kappa(\delta)}{\delta} \quad \text { and } \quad g_{2}(\delta)=\mathcal{H} \kappa(\delta) \frac{\delta^{r}}{2^{r}(r+1)}
$$

Then,

$$
\|\Phi(t)\|_{2} \leq \kappa(\delta) \mathrm{e}^{\left(\alpha+g_{2}(\delta)\right) t}\|\Phi(0)\|_{2}
$$

For $m \delta \leq t \leq(m+1) \delta$, we get

$$
\begin{aligned}
\|\Phi(t)\|_{2} & \leq \kappa(\delta)^{m+1} \mathrm{e}^{\left(\alpha+g_{2}(\delta)\right) t}\|\Phi(0)\|_{2} \\
& =\kappa(\delta) \mathrm{e}^{g_{1}(\delta) m \delta+\left(\alpha+g_{2}(\delta)\right) t}\|\Phi(0)\|_{2} \\
& \leq \kappa(\delta) \mathrm{e}^{\left(\alpha+g_{1}(\delta)+g_{2}(\delta)\right) t}\|\Phi(0)\|_{2} .
\end{aligned}
$$

If $\xi_{0}=0$, then we have

$$
\lim _{\delta \rightarrow 0^{+}} g_{1}(\delta)+g_{2}(\delta)=0 \text { and } \quad \lim _{\delta \rightarrow \infty} g_{1}(\delta)+g_{2}(\delta)=\infty .
$$

Thus, given $\eta>0$, there exists $\delta>0$ such that $g_{1}(\delta)+g_{2}(\delta)=\eta$, and the first claim follows.

If $\xi_{0}>0$, let $h(\delta)=g_{1}(\delta)-g_{2}(\delta)$, for $\delta>0$ and $h(0)=\xi_{0}$; then $h$ is continuous, and we have $h(0)>0$, and $\lim _{\delta \rightarrow \infty} h(\delta)=-\infty$. Hence there exists $\delta^{*}>0$ such that $g_{1}\left(\delta^{*}\right)=g_{2}\left(\delta^{*}\right)$; then let

$$
\bar{\delta}=\arg \min _{\delta \in[0, \delta *]} g_{1}(\delta)+g_{2}(\delta), \quad \rho=g_{1}(\bar{\delta})+g_{2}(\bar{\delta}),
$$

and we obtain the second case.

Remark 1. The proof of Theorem 2 shows that in the second, and more common case, we can always take $\left.\rho(\delta)=2 \max \left\{g_{1}(\delta), g_{2}(\delta)\right)\right\}$, which is usually easier to compute, but does not yield an optimal bound.

Remark 2. Theorem 2 generalizes the corresponding result in Coppel (1978, Proposition 4, page 5) in two ways: by allowing $M(t)$ to be less regular-Holder of degree $r$ instead of Lipschitz - and by allowing the pre-factor in the exponential to be an arbitrary continuous function of $t$ with at most polynomial growth.

4.3. Bounds for matrix exponential. In order to apply Theorem 2, we need a bound on the exponential of $M$ with the coefficients frozen in time. If the spectral abscissa of $M$ is known, then it remains to obtain a good estimate for $\kappa(t)$ to complete the bound. For general matrices, a particular set of inequalities can be found in Kågström (1977), where the spectral norm is used. For $2 \times 2$ or $3 \times 3$ matrices, the following bounds provide better estimates: 
Proposition 1. Let $M \in \mathcal{M}_{2}(\mathbb{R})$, such that $\sigma(M)=\left\{\lambda_{1}, \lambda_{2}\right\} \subset \mathbb{R}$, with $\lambda_{1} \geq \lambda_{2}$. Then

$$
\left\|\mathrm{e}^{t M}\right\|_{2} \leq \mathrm{e}^{\lambda_{1} t}\left(1+F(t)\left\|M-\lambda_{1} I\right\|_{F}\right) .
$$

with

$$
F(t)= \begin{cases}\frac{1-\mathrm{e}^{\left(\lambda_{2}-\lambda_{1}\right) t}}{\lambda_{1}-\lambda_{2}}, & \lambda_{1} \neq \lambda_{2} \\ t, & \lambda_{1}=\lambda_{2}\end{cases}
$$

where $d=\lambda_{2}-\lambda_{1}$.

Proof. The bound follows from the expression

$$
\mathrm{e}^{t M}=a(t) M+b(t) I, \quad a(t)=\frac{\mathrm{e}^{\lambda_{1} t}-\mathrm{e}^{\lambda_{2} t}}{\lambda_{1}-\lambda_{2}}, \quad b(t)=\mathrm{e}^{\lambda_{1} t}-a(t) \lambda_{1} .
$$

A very similar proof yields also the following result:

Proposition 2. Let $M \in \mathcal{M}_{3}(\mathbb{R})$, such that $\sigma(M)=\left\{\lambda_{1}, \lambda_{2}, \lambda_{3}\right\} \subset \mathbb{R}$, with $\lambda_{1}>\lambda_{2}>$ $\lambda_{3}$. Then

$$
\left\|\mathrm{e}^{t M}\right\|_{2} \leq \mathrm{e}^{\lambda_{1} t}\left(1+\sqrt{2}\left(b(t)\|N\|_{F}+a(t)\left\|N^{2}\right\|_{F}\right)\right),
$$

where

$$
N=M-\lambda_{1} I, \quad a(t)=\frac{G_{1}(t)-G_{2}(t)}{d_{2}-d_{1}}, \quad b(t)=G_{1}(t)+d_{1} a(t) ;
$$

with $d_{1}=\lambda_{1}-\lambda_{2}, d_{2}=\lambda_{1}-\lambda_{3}$, and also:

$$
G_{1}(t)=\frac{1-\mathrm{e}^{-d_{1} t}}{d_{1}} \quad \text { and } \quad G_{2}(t)=\frac{1-\mathrm{e}^{-d_{2} t}}{d_{2}} .
$$

4.4. Revisiting generalised SIR. Recall that System (5) is of the type described by Equation (1), in particular we have that

$$
A=\left(\begin{array}{cc}
-\mu_{h} & 0 \\
0 & -\left(\gamma+\mu_{h}\right)
\end{array}\right), G(y)=\left(\begin{array}{cc}
-g(y) & 0 \\
g(y) & 0
\end{array}\right), b^{T}=\left(\mu_{h}, 0\right) \text {, and } C=\left(\begin{array}{ll}
0 & 1
\end{array}\right) \text {. }
$$

Therefore, since the second alternative observer is in class described by System (14), the error equation is given by

$$
\dot{e}=M(y) e, \quad M(y)=\left(\begin{array}{cc}
-\mu_{h}-g(y) & -k_{1} \\
g(y) & -\left(\gamma+\mu_{h}\right)-k_{2}
\end{array}\right) .
$$

It is easy to check that System 5 is parametrically linearly observable.

We will now verify that we can achieve arbitrary exponential convergence of the observer. 
In order to do so, we first observe that, since System (5) is parametrically linearly observable, we are able to choose $K(y)$ such that the spectra of $M(y)$ is prescribed. In particular, if we set

$$
k_{1}(y)=\frac{c^{2} \phi_{1}\left(\phi_{1}+\phi_{2}\right)}{y \hat{g}(y)} \quad \text { and } \quad k_{2}(y)=c\left(2 \phi_{1}+\phi_{2}\right)+g(y)-\gamma
$$

with $\phi_{1}, \phi_{2}>0$, and where $\hat{g}(y)=\frac{g(y)}{y}$, then we obtain that $\sigma(M(y))=\left\{-\mu_{h}-g(y)-\right.$ $\left.c \phi_{1},-\mu_{h}-g(y)-c\left(\phi_{1}+\phi_{2}\right)\right\}$. In this case, Proposition 1 yields for fixed $y>0$ :

$\left\|\mathrm{e}^{t M}\right\|_{2} \leq \mathrm{e}^{-\left(\mu_{h}+g(y)+c \phi_{1}\right) t} \kappa(t), \quad \kappa(t)=1+\frac{1-\mathrm{e}^{-c \phi_{2} t}}{c \phi_{2}} \mathcal{N}, \quad \mathcal{N}=\left\|M+\left(\mu_{h}+c \phi_{1}+g(y)\right) I\right\|_{F}$.

By Theorem 2, there exists $\delta$ such that $\|\Phi(t)\|_{2} \leq \kappa(\delta) \exp ((\alpha+\rho) t)$ with $\rho=\frac{\log \kappa(\delta)}{\delta}+$ $\frac{\mathcal{H}}{4} \delta \kappa(\delta)$. Here $\alpha=-\left(\mu_{h}+g(y)+c \phi_{1}\right)$.

In addition, if $c \phi_{2} \gg 1$, we also have

$$
\mathcal{N}=\frac{c^{2} \phi_{1}\left(\phi_{1}+\phi_{2}\right)}{y \hat{g}}+\frac{\left(\phi_{1}^{2}+\left(\phi_{1}+\phi_{2}\right)^{2}\right)}{2 \phi_{1}\left(\phi_{1}+\phi_{2}\right)} y \hat{g}+\mathcal{O}\left(c^{-2}\right) .
$$

In what follows, we now take $\phi_{2}=1$, and $0<\phi_{1} \leq 1$, just for the sake of simplicity. Assuming $\delta c \gg 1$, we then find

$$
\begin{aligned}
\delta \kappa(\delta) & =\delta\left[1+\left(1-\mathrm{e}^{-c \delta}\right) \frac{\mathcal{N}}{c}\right] \\
& \leq \delta\left(1+\frac{\mathcal{N}}{c}\right) \\
& =\delta \frac{c+\mathcal{N}}{c} .
\end{aligned}
$$

and

$$
\begin{aligned}
\frac{\log \kappa(\delta)}{\delta} & =\delta^{-1} \log \left[1+\left(1-\mathrm{e}^{-c \delta}\right) \frac{\mathcal{N}}{c}\right] \\
& \leq \delta^{-1} \log \left(1+\frac{\mathcal{N}}{c}\right) .
\end{aligned}
$$

Then $\frac{\mathcal{H}}{4} \delta \kappa(\delta)$ and $\delta^{-1} \log \kappa(\delta)$ will balance if we choose

$$
\delta=\frac{2}{\mathcal{H}^{1 / 2}}\left(\frac{c}{c+\mathcal{N}} \log \left(1+\frac{\mathcal{N}}{c}\right)\right)^{1 / 2} .
$$


In this case, since $0<\phi_{1} \leq 1$, we obtain

$$
\begin{aligned}
\rho(\delta) & \leq \delta \kappa(\delta)+\delta^{-1} \log \delta \\
& \leq \mathcal{H}^{1 / 2}\left[\left(1+\frac{\mathcal{N}}{c}\right) \log \left(1+\frac{\mathcal{N}}{c}\right)\right]^{1 / 2} \\
& \leq \mathcal{H}^{1 / 2}\left(\frac{2 \phi_{1}}{y \hat{g}(y)}\right)^{1 / 2} c^{1 / 2}\left(\log c+\log \frac{2 \phi_{1}}{y \hat{g}(y)}\right)^{1 / 2}+\mathcal{O}\left(\left(\frac{\log c}{c}\right)^{1 / 2}\right) \\
& \leq \mathcal{H}^{1 / 2}\left(\frac{2}{\hat{g}(y)}\right)^{1 / 2} c^{1 / 2}\left(\log c+\log \frac{2 \phi_{1}}{y \hat{g}(y)}\right) .
\end{aligned}
$$

In the typical applications, we have that System (5) is uniformly persistent, and hence we can assume that $0<\bar{y} \leq y \leq 1$, together with $\frac{\phi_{1}}{y} \leq \frac{1}{\bar{y}}$. Hence

$$
\begin{aligned}
-c \phi_{1}+\rho(\delta) & \leq-c \phi_{1}\left(1-\mathcal{H}^{1 / 2}\left(\frac{2}{g(y)}\right)^{1 / 2} c^{-1 / 2}\left(\log c+\log \frac{2 \phi_{1}}{y \hat{g}(y)}\right)\right) \\
\leq & -c \phi_{1}\left(1-\left(\frac{2 \mathcal{H}}{m}\right)^{1 / 2} c^{-1 / 2}\left(\log c+\log \frac{2}{\bar{y} m^{\prime}}\right)\right) .
\end{aligned}
$$

where $m=\min _{y \in[\bar{y}, 1]} y g(y)>0$ and $m^{\prime}=\min _{y \in[\bar{y}, 1]} \hat{g}(y)>0$.

Therefore, we have $-c \phi_{1}+\rho(\delta)<0$ provided $c$ is sufficiently large.

More precisely, let

$$
\Lambda=-\left(\frac{m}{8 \mathcal{H}}\right)^{1 / 2} \exp \left(-\frac{1}{\bar{y} m^{\prime}}\right) .
$$

Then, we have that $-c \phi_{1}+\rho(\delta)<0$, provided that we have both

$$
\begin{aligned}
& c>\exp \left(-2 W_{k}(\Lambda)\right), \quad k= \begin{cases}0 & \Lambda \geq 1, \\
-1 & \Lambda \leq 1 ;\end{cases} \\
& c \gg M \frac{9 \bar{y}}{\sqrt{2}}, \quad M=\max \hat{g}(y),
\end{aligned}
$$

and where $W_{0}$ and $W_{-1}$ are the principal and secondary branches, respectively, of the W-Lambert function.

4.5. Some experiments. In order to test the efficiency of our proposed observer (12) we performed a batch of numerical experiments. Numerical simulations showing the performance of the observer given by (12) can be seen in Figure 1 and in Figure 2 - in the latter, the output $y(t)$ is corrupted by white noise. Convergence of the estimator was quite fast and the estimation was quite robust to the addition of noise.

The corresponding simulations for the high-gain observer (8)-with the same initial conditions and the same noise - are given in Figure 3. It can be noticed that the highgain observer converges faster than the observer (12) but it is more sensitive to noisy data. 


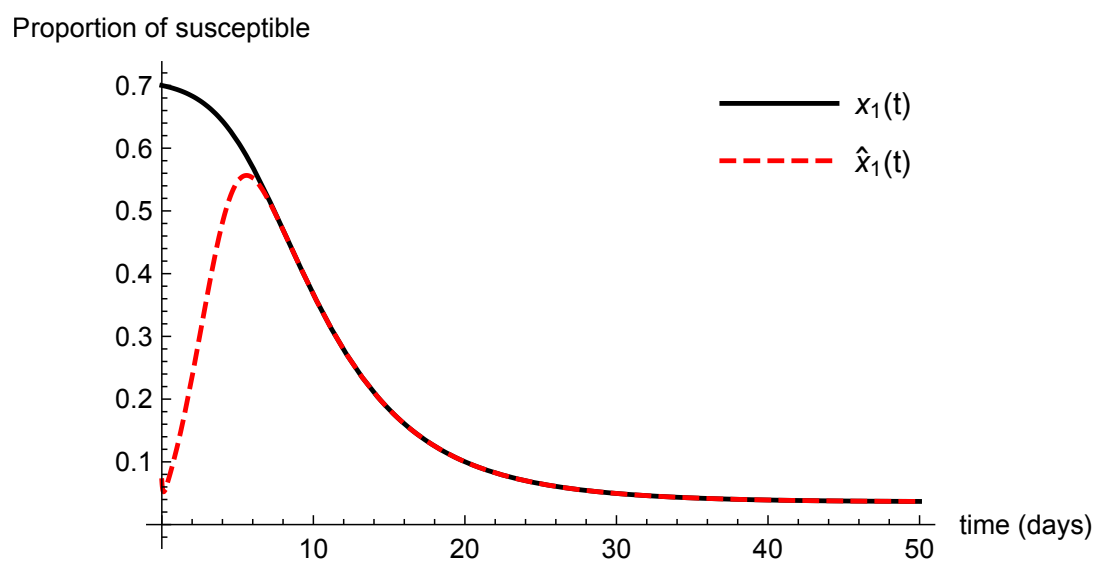

FiguRe 1. Simulation of system (2) and its estimator (12) where $k_{1}$ and $k_{2}$ are given by (19) with $\phi_{2}=1$, and $\phi_{1}=y(t)$ : the solid curve displays the evolution of $x_{1}(t)$ (proportion of susceptible individuals), while the dashed curve displays its estimate $\hat{x}_{1}(t)$ delivered by the observer (12). Convergence of $\hat{x}_{1}(t)$ towards $x_{1}(t)$ is quite fast as expected.

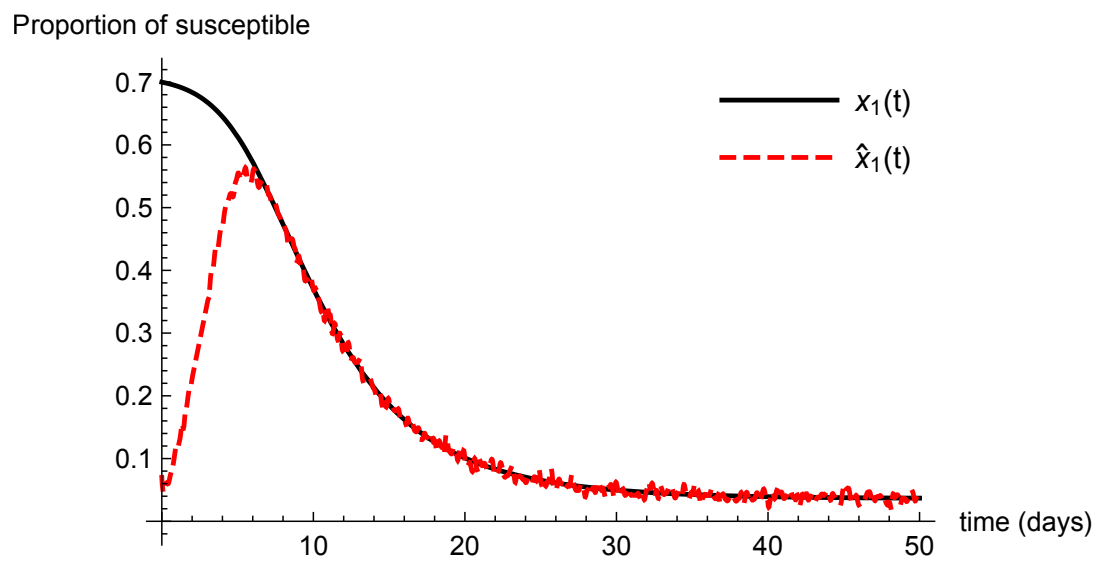

Figure 2. Simulation of system (2) and its estimator (12) where $k_{1}$ and $k_{2}$ are given by (19) with $\phi_{2}=1$, and $\phi_{1}=y(t)$, the output $y(t)$ is corrupted by white noise: the solid curve displays the evolution of $x_{1}(t)$ (proportion of susceptible individuals), while the dashed curve displays its estimate $\hat{x}_{1}(t)$ delivered by the observer (12). Convergence of $\hat{x}_{1}(t)$ towards $x_{1}(t)$ is quite fast as expected. 
Proportion of susceptible

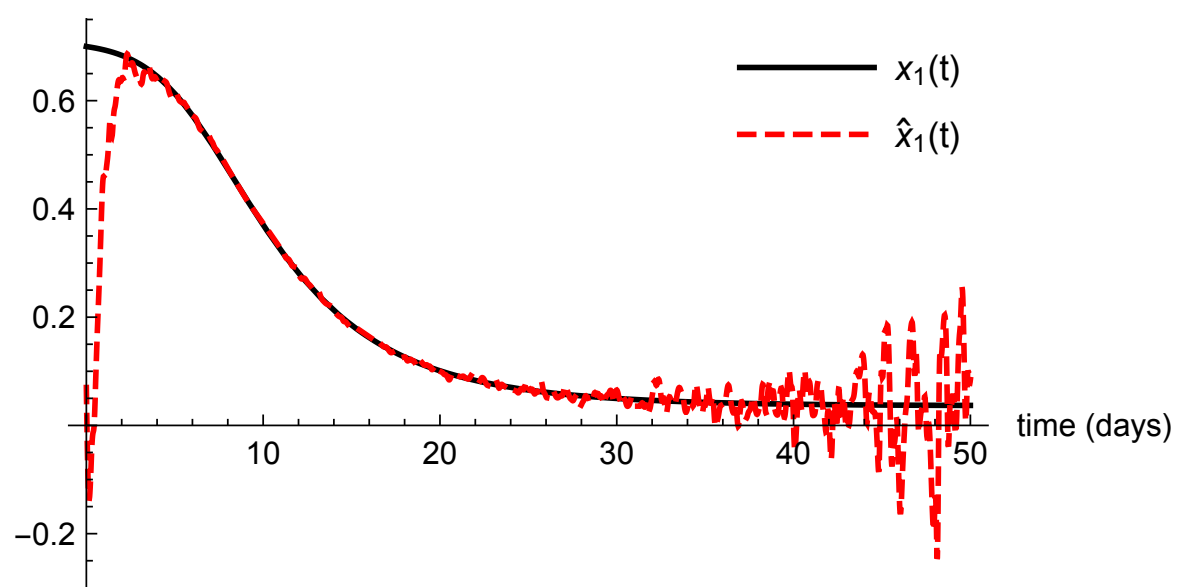

Figure 3. Simulation of system (2) and the high-gain observer (8), the output $y(t)$ is corrupted by white noise: the solid curve displays the evolution of $x_{1}(t)$, while the dashed curve displays its estimate $\hat{x}_{1}(t)$ delivered by the observer (12). Convergence of $\hat{x}_{1}(t)$ towards $x_{1}(t)$ is quite fast as expected but this estimator is quite sensitive to noisy measurements

\section{An APPLiCATion to a REDUCED ARbovirus MODEL}

5.1. The reduced Bailey-Dietz arboviruse model. The classical Bailey-Dietz (Bailey, 1975; Dietz, 1975) model in adimensional variables - cf. Souza (2014); Esteva and Vargas (1998) - is given by

$$
\begin{aligned}
& \dot{x}_{1}=\mu_{h}\left(1-x_{1}\right)-\delta x_{1} x_{3} \\
& \dot{x}_{2}=\delta x_{1} x_{3}-\left(\mu_{h}+\gamma\right) x_{2} \\
& \dot{x}_{3}=\sigma x_{2}\left(1-x_{3}\right)-\mu_{v} x_{3}
\end{aligned}
$$

Here $x_{1}$ represents the fraction of susceptible hosts, $x_{2}$ the fraction of infected hosts, and $x_{3}$ the fraction of infected vectors. The basic reproduction number is given by $R_{0}^{2}=\frac{\delta \sigma}{\left(\gamma+\mu_{h}\right) \mu_{v}}$. This model has been extensively used to describe dengue dynamicse.g. Nishiura (2006); Esteva and Vargas (1998). Under reasonable hypothesis - see the discussion in Souza (2014) - we can consider the so-called fast vector regime by assuming that $\sigma=\epsilon^{-1} \bar{\sigma}$ and $\mu_{v}=\epsilon^{-1} \bar{\mu}_{v}$-but see also Rocha et al. (2013) for alternative scalings.

It can be reduced to the $2 \times 2$ model (2) mentioned in Section 2 by means of a multiscaling argument - cf. Souza (2014), but see also a more informal derivation on Keeling 
and Rohani (2008). For convenience, we recall that it is given by

$$
\left\{\begin{array}{l}
\dot{x}_{1}=\mu_{h}\left(1-x_{1}\right)-f\left(x_{2}\right) x_{1}, \\
\dot{x}_{2}=-\left(\gamma+\mu_{h}\right) x_{2}+f\left(x_{2}\right) x_{1} \\
x_{3}=f\left(x_{2}\right)
\end{array}\right.
$$

with $f(x)=\frac{\delta \sigma x}{\mu_{v}+\sigma x}$. A noticeable fact is that the $R_{0}$ for the reduced system is the same as the on for the full system.

We have assumed that the measurable output was $y=x_{2}$. However, as we shall see below, in typical applications this assumption is not quite correct, and the raw data should be processed before it can be feed into the observer.

5.2. Measuring the output. For the numerical experiments we use dengue data from the city of Rio de Janeiro (Brazil) from the years 2000 through 2008-more precisely the data series starts on the January 2000 and goes until June 2008. This data was taken from the database of notified dengue cases in the district of Jacarepaguá, in the city of Rio de Janeiro and an excerpt of it can be found in Table 1 in Appendix A.

For this dataset, the only available information is the number of reported cases over a month. This needs to be converted to the number of infectives which was the output assumed to be available. This is will be done by augmenting the data as follows: First we recall that, under the reduced Bailey-Dietz model, infectives arise at a rate of $f\left(x_{2}\right) x_{1}$. Hence, the new cases are given by

$$
\mathrm{NC}(t ; t+T)=\int_{t}^{t+T} f\left(x_{2}(s)\right) x_{1}(s) \mathrm{d} s .
$$

On the other hand, let us write $F(t)=f\left(x_{2}(t)\right) x_{1}(t)$, and on using the second equation in System (20) we obtain

$$
x_{2}(t)=x_{2}\left(t_{0}\right) \mathrm{e}^{-\left(\mu_{h}+\gamma\right)\left(t-t_{0}\right)}+\int_{t_{0}}^{t} \mathrm{e}^{-\left(\mu_{h}+\gamma\right)(t-s)} F(s) \mathrm{d} s .
$$

In the absence of further information, we then assume the time of infection to be uniformly distributed over the observation period. This amounts to chose $F$ constant over each such a period, and if we write $\Delta t$ to denote the length of the observation period we obtain:

$$
\mathrm{NC}(t ; t+\Delta t)=F(t) \Delta t .
$$

For the sake of simplicity, let us assume uniform observation periods, and write $t_{k}=$ $t_{0}+k \Delta t$ for the observation dates. In this case, if $t \in\left[t_{k}, t_{k+1}\right)$, we then arrive at

$$
\begin{aligned}
x_{2}(t)= & x_{2}\left(t_{0}\right) \mathrm{e}^{-\left(\mu_{h}+\gamma\right)\left(t-t_{0}\right)}+\frac{\mathrm{e}^{\left(\mu_{h}+\gamma\right) \Delta t}-1}{\left(\mu_{h}+\gamma\right) \Delta t} \sum_{j=0}^{k-1} \mathrm{NC}\left(t_{j} ; t_{j+1}\right) \mathrm{e}^{-\left(\mu_{h}+\gamma\right)\left(t-t_{j}\right)}+ \\
& \frac{\mathrm{NC}\left(t_{k} ; t_{k+1}\right)}{\left(\mu_{h}+\gamma\right) \Delta t}\left(1-\mathrm{e}^{-\left(\mu_{h}+\gamma\right)\left(t-t_{k}\right)}\right) .
\end{aligned}
$$


The above expression provides a simple, yet effective estimate of the infective population for the dataset considered. For alternative approaches see Cauchemez and Ferguson (2008); Finkenstädt and Grenfell (2000).

In the inset of Figure 4 we can see a subset of this data, together with the corresponding estimated number of infected individuals indicated by the continuous black curve.

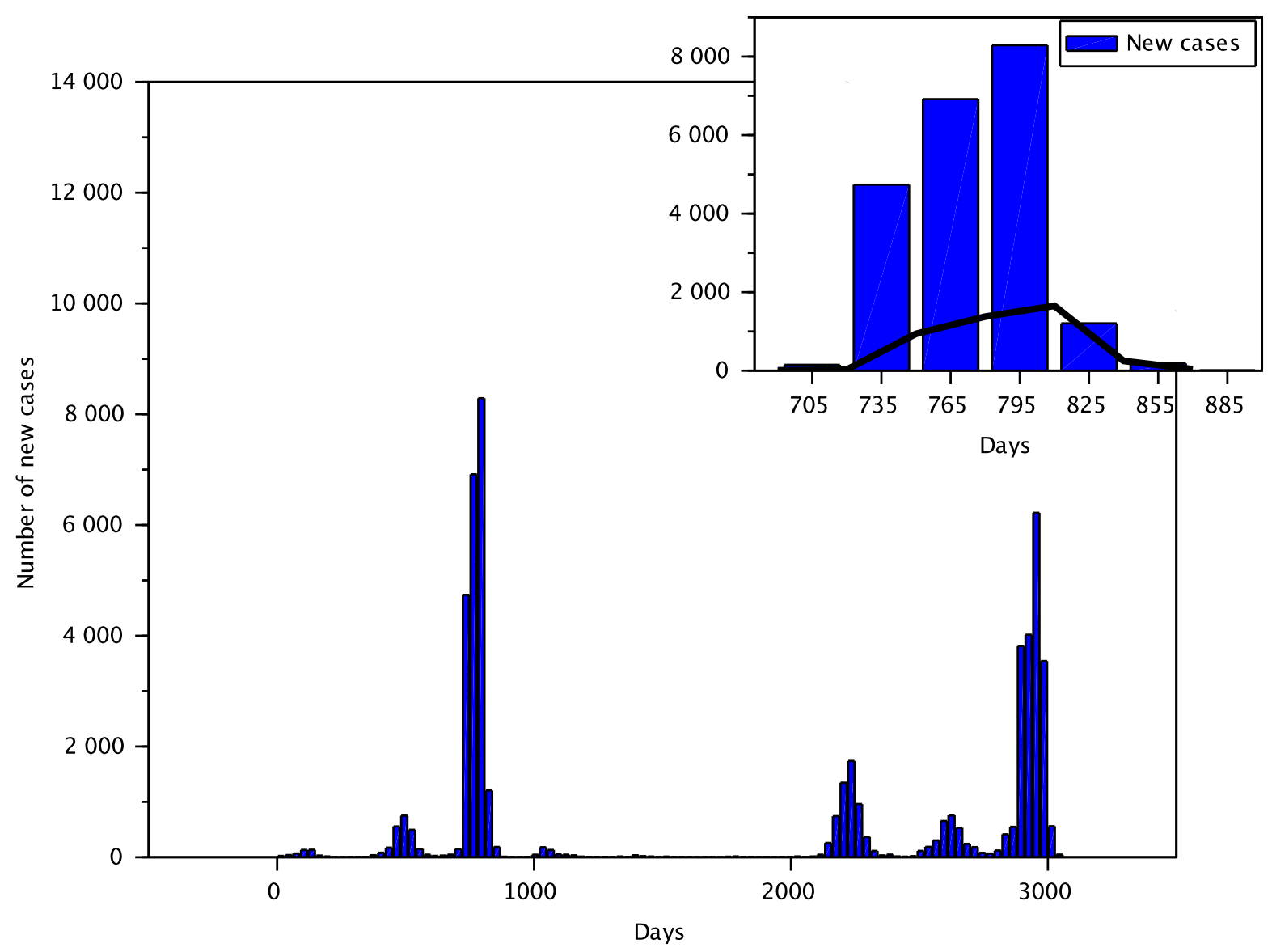

FiguRE 4. Notified cases per month from Jan 2000 through June 2008 in the district of Jacarepaguá, in the city of Rio de Janeiro. Inset: Zoom view of the period from November 2001 to May 2002, where there has been on of the greatest epidemics of dengue in Rio. The bars are centred in the middle of the corresponding month reported. The continuous black curve indicate the number of infective individuals obtained using the estimation procedure described in Section 5.2. 


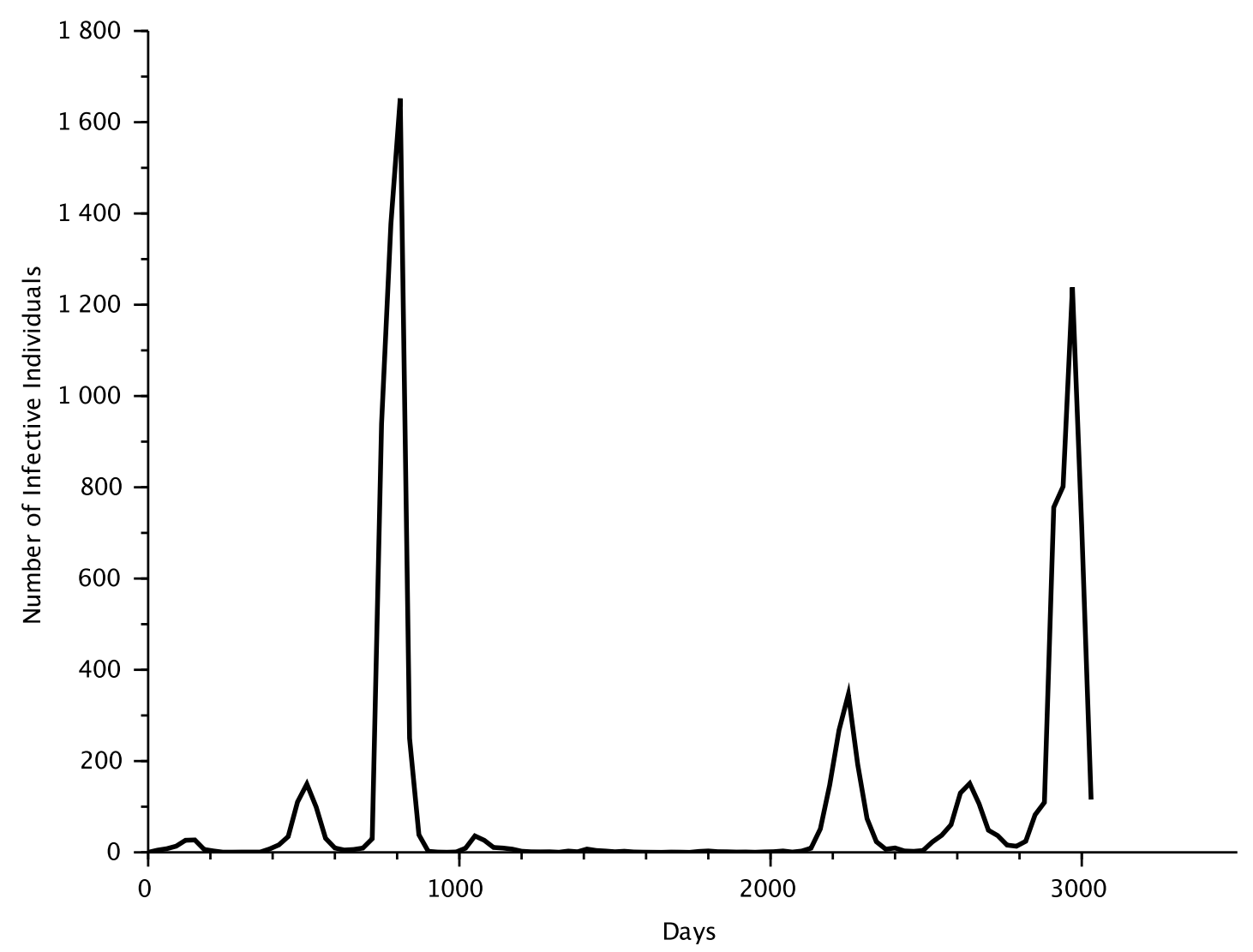

Figure 5. Number of infective individuals obtained from the available data on the number of new cases, along the time period of the dataset considered.

5.3. The observer in action. We have then applied the observer described in Section 4 to the System (20) in order to estimate the proportion of susceptible population. For the results presented here, we used the following gains:

$$
\begin{aligned}
& k_{1}(y)=\left(\frac{c}{\max (y, \epsilon)}-\hat{g}(y)\right)\left(d+\left(\frac{c}{\max (y, \epsilon)}-(g \hat{)}(y)) y\right) \frac{1}{\hat{g}(y)}\right. \\
& k_{2}(y)=d+2 \frac{c y}{\max (y, \epsilon)}-g(y)-\gamma,
\end{aligned}
$$

where $c, d$ and $\epsilon$ are positive constants - for the results shown typical choices were $c=3, d=1$ and $\epsilon=10^{-4}$. The gains given above in (21) provided a faster convergence than the ones given in (19) for this data set. In general, for a given set of data, different 
variations might yield faster convergence although numerical experiments suggest that the gains in (19) always yield a reasonably fast convergence.

The proportion of susceptible individuals is shown in Figure 6. In order to understand the difference between the two pictures, we introduce the following definition: an initial condition for the observer given by Equation (14) is consistent, if $y(0)$ is the same as the one used for reconstructing $y$ from the data; otherwise we will say that it is inconsistent. Thus, in Figure 6 show estimates for three initial conditions for the proportion of susceptible, with different choices of initial conditions for the proportion of infective: In Figure 6(A) we see the trajectory of the estimation of the susceptible class for a set of consistent initial conditions, while in Figure 6(B) we see trajectories with inconsistent initial conditions. Notice that even when the initial condition is inconsistent, the observer eventually converges to the correct behaviour within a time frame of two months. Notice, however, that convergence is much faster when the initial condition is consistent.

The complete trajectory for one consistent choice of initial condition can be seen in Figure 7:

Convergence of the observer is confirmed in Figure 8, where the trajectory of the observer for a consistent initial condition is shown together with the corresponding data estimated trajectory for the measured input:

Finally, Figure 9 display the complete picture. Notice that the result obtained from the estimation strongly suggests that the population in this region has been already exposed to the dengue virus before. 


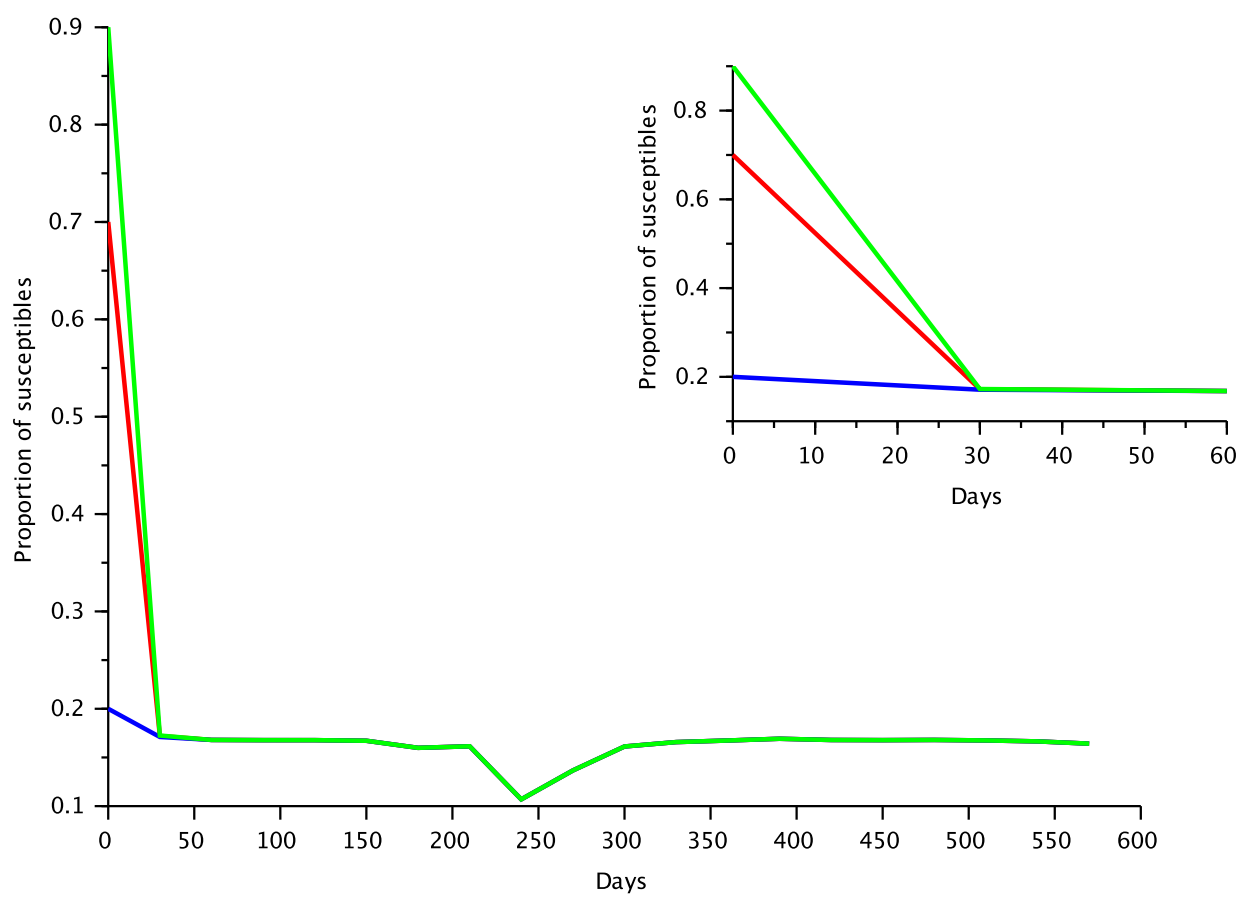

(A)

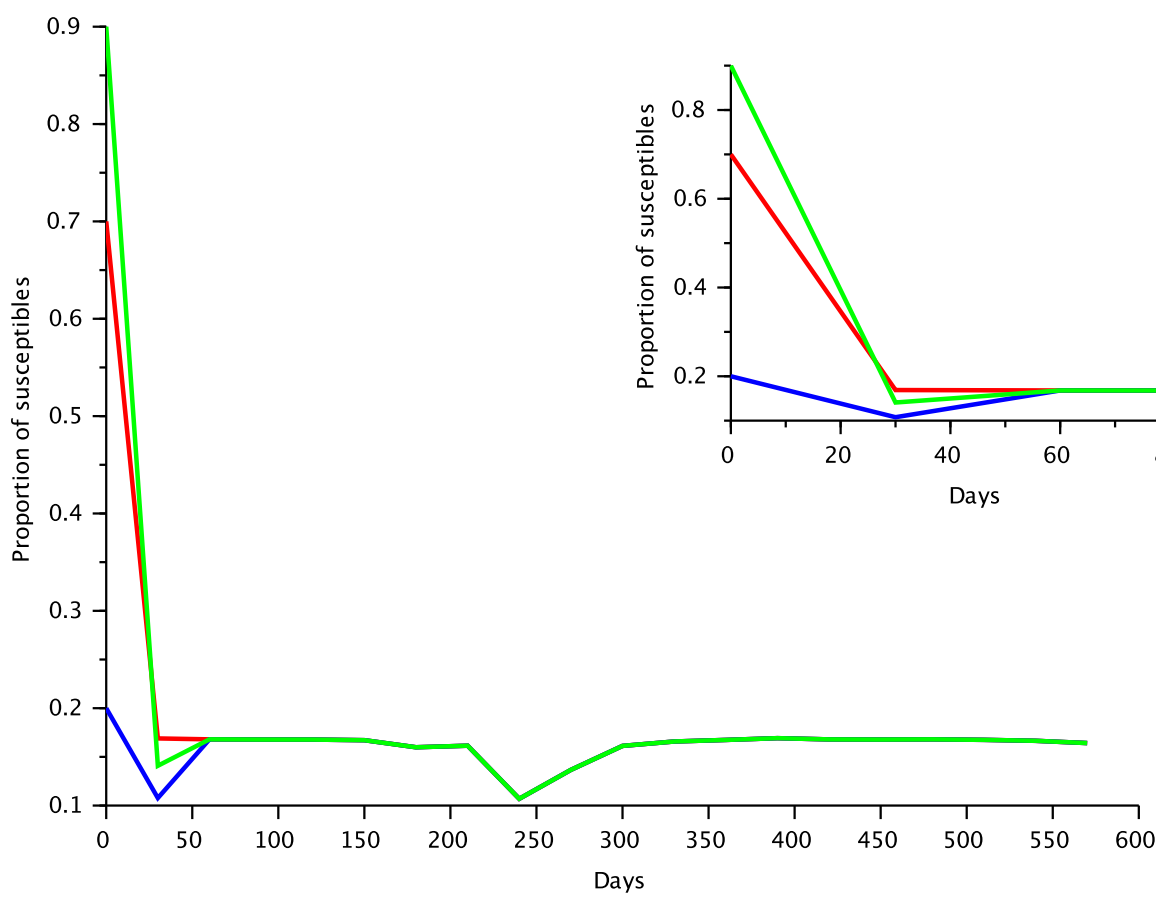

(B)

FIGURE 6. Estimates of the proportion of susceptible individuals for three different choices of initial conditions for the proportion of susceptible and with (A) consistent initial conditions for the proportion of infective; and (B) non-consistent initial conditions. Insets: Zoom showing earlier times of the trajectories. 


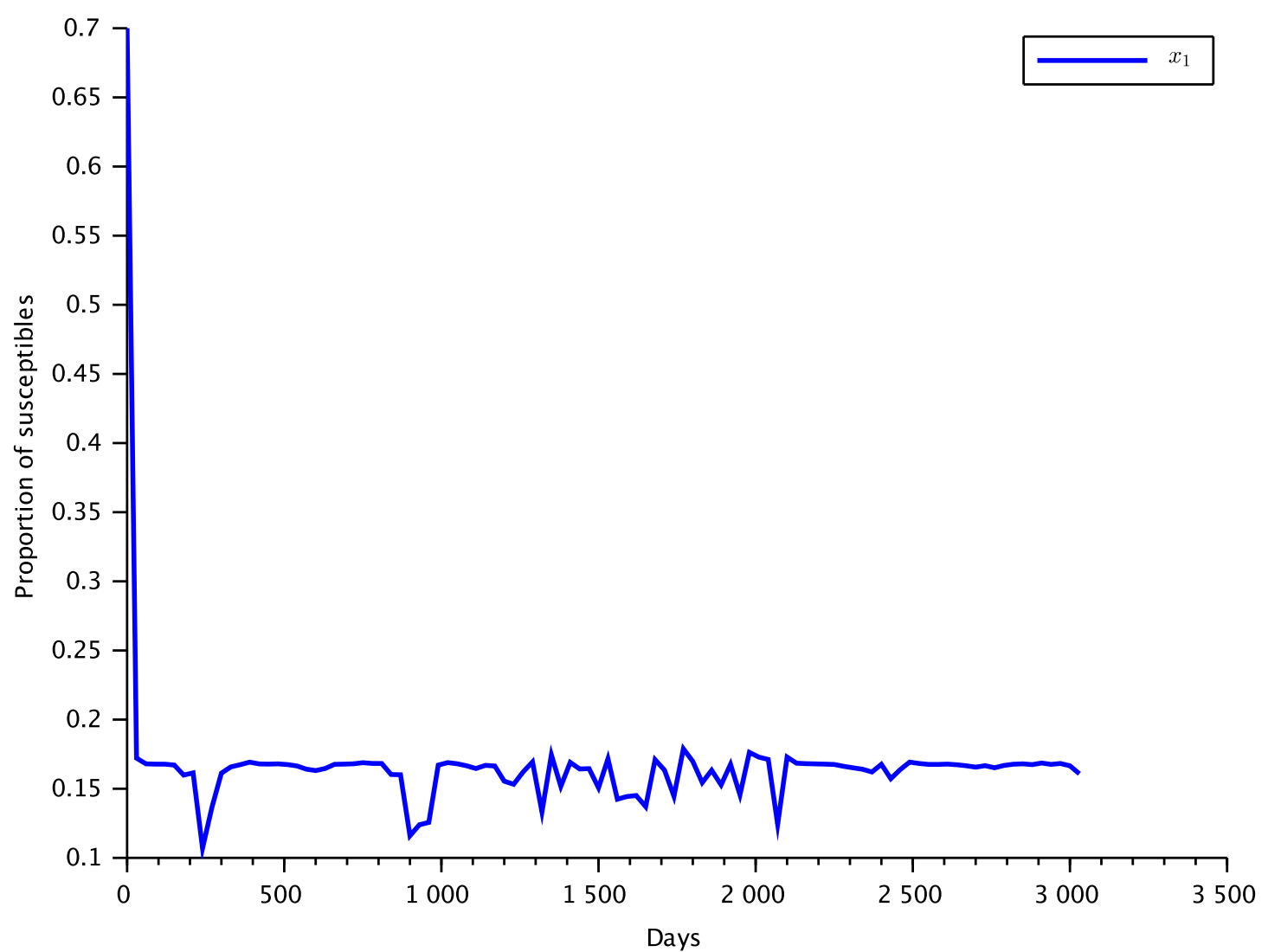

FigURE 7. Estimate of the susceptible proportion of individuals over the considered time-frame. 


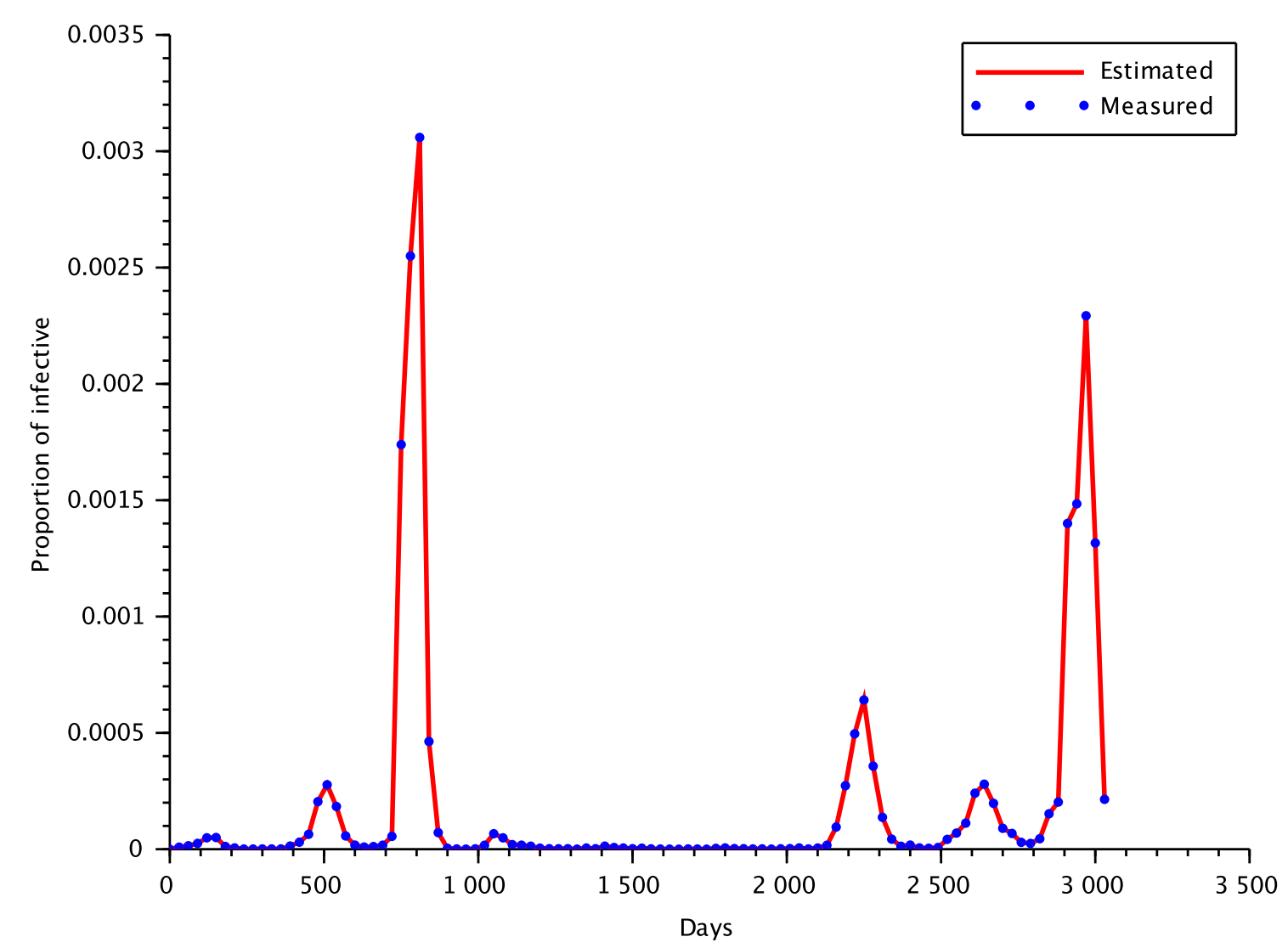

FiguRE 8. Proportion of infective individuals obtained from field data as discussed in Section 5.2 - and the corresponding observer estimates. As expected, convergence of the estimator is quite fast, and the observer trajectory tracks the discrete data quite accurately. 


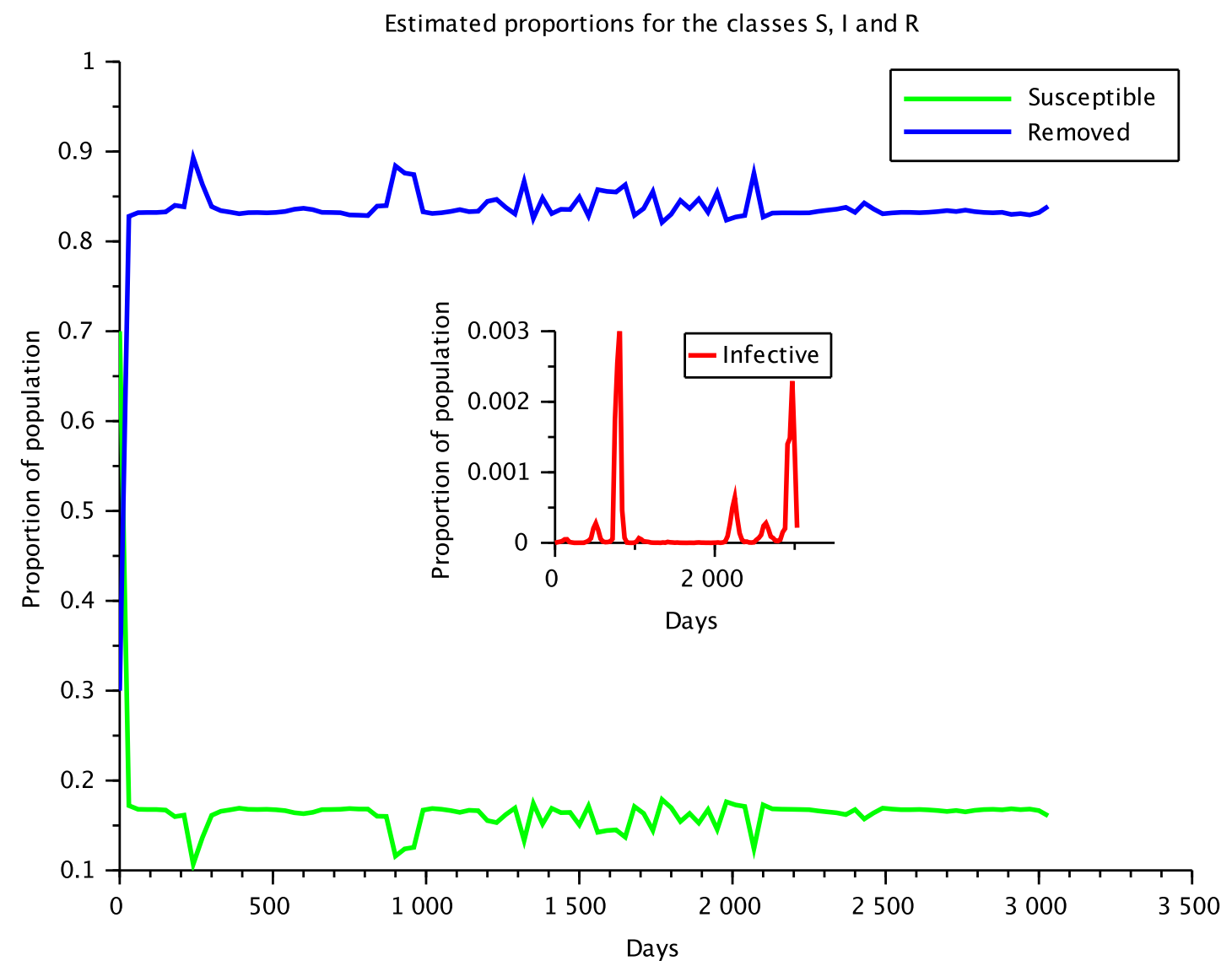

Figure 9. The full picture: in the main figure we have estimates for the susceptible and removed classes. In the inset, we show the estimate for the infective class - which is in a much smaller scale. This picture suggests that the population of the district of Jacarépaguá has been exposed to the dengue virus and it should be mainly seropositive. 


\section{Discussion}

In this work we considered the class of epidemiological models of the form given in Equation 1, which includes most of the well known epidemiological models for directly transmitted diseases. For this class, we have proposed a simple and easy implementable observer given by Equation (14). This proposed observer leads to a non-autonomous error equation, and this renders the error analysis considerably more difficult. We overcame this problem by obtaining a novel bound for fundamental matrices - a slight generalisation of the classical bound by Coppel (1978), which nevertheless turns out to be crucial for such bound to be usable for the error analysis.

Optimal bounds, within the framework of Theorem 2, can be obtained numerically, but suboptimal bounds can be obtained analytically. Using such bounds, we were able to show that, when the observer is applied to the generalised SIR model, we can obtain arbitrarily exponential convergence by choosing the tuning gains (here $k_{1}$ and $k_{2}$ ) to be sufficiently large.

We then considered the reduced Bailey-Dietz model studied in Souza (2014), which is a special case of the generalised SIR model, and constructed the proposed observer for this reduced system. This observer was applied with real data coming from the district of Jacarepaguá in the city of Rio de Janeiro to estimate the susceptible population in this area and, as indicated by the analysis in Section 4, the estimate converges very fast towards the population state - this can also be seen graphically in Figure 6(B), where simulations with three different arbitrary initial conditions are displayed, and the corresponding estimates become visually indistinguishable after a short time. The typical values of the tuning gains that yielded such an exponential convergence were usually somewhat smaller that the values obtained from the theory - which is to be expected, given that suboptimal bounds were used in the analysis.

Although not presented here, the approach can be extended to other models. For instance, when immunity to the disease is temporary, an appropriate alternative model is SIRS, and is given by

$$
\left\{\begin{aligned}
\dot{S} & =\mu(N-S)-g(I) S+\xi R \\
\dot{I} & =g(I) S-(\mu+\gamma) I \\
\dot{R} & =\gamma I-(\mu+\xi) R
\end{aligned}\right.
$$

Recall that for the generalised SIR, the decay estimate has the form

$$
-c \bar{y}\left(1-C_{1} c^{-1 / 2}\left(\log c+\log C_{2}\right)^{1 / 2}\right),
$$

where $c$ is a parameter that can be taken arbitrarily large, and hence arbitrary exponential convergence can be attained, at least theoretically. If we apply the observer assuming only that the infectious individuals are measured, and perform an analogous analysis - but now using Proposition 2-we obtain an estimate of the form:

$$
-c \bar{y}\left(1-C_{1}\left(\log c+\log C_{2}\right)^{1 / 2}\right),
$$

which is not sufficient to yield the same behaviour. There are a number of ways to circumvent this issue: (i) use a judicious choice of gains to obtain exponential convergence 
of order $\mu+\xi$, which will be fine if $\xi$ is not small; (ii) estimate the number of removed individuals from raw data - using a similar approach to that was used to estimate the number of infectious in Section 5.2-and rework the analysis performed in Section 4.4 taking into account that $C$ is now a $2 \times 3$ matrix, and that gain matrix $K(y)$ is now $3 \times 2$; in this case, the extra freedom in $K(y)$ allow us to recover an estimate of the form given in Equation (23), and hence exponential convergence is retained.

We hope that such an easily implementable observer might contribute to an increasing use of such tools in epidemiology. The possible applications are countless and range from estimation of the susceptible population to model calibration. From the practical point of view, one rather interesting feature of the observer proposed here, and of the companion error analysis, is that both can be applied to non-autonomous models. In the future, we hope to obtain a more systematic tuning of the observers for different models.

\section{REFERENCES}

A. Abdelhedi, D. Boutat, L. Sbita, and R. Tami. Extended observer to estimate the spreading of contagious disease. In 2014 European Control Conference (ECC), pages 1843-1848, June 2014. doi: 10.1109/ECC.2014.6862172.

A. Abdelhedi, D. Boutat, L. Sbita, R. Tami, and D.-Y. Liu. Observer design for a class of nonlinear piecewise systems. Application to an epidemic model with treatment. Math. Biosci., 271:128-135, 2016.

S. Alonso-Quesada, M. De la Sen, R. P. Agarwal, and A. Ibeas. An observer-based vaccination control law for an SEIR epidemic model based on feedback linearization techniques for nonlinear systems. Adv. Difference Equ., pages 2012:161, 32, 2012.

J. Alvarez-Ramirez, M. Meraz, and J. X. Velasco-Hernandez. Feedback control of the chemotherapy of HIV. International Journal of Bifurcation and Chaos, 10(09):22072219, 2000. doi: 10.1142/S0218127400001377. URL https://www . worldscientific . com/doi/abs/10.1142/S0218127400001377.

R. M. Anderson and R. M. May. Infectious Diseases of Humans. Dynamics and Control. Oxford science publications, 1991.

J. Arino and P. van den Driessche. A multi-city epidemic model. Math. Popul. Stud., 10(3):175-193, 2003. doi: 10.1080/08898480306720.

M. S. Aronna and P.-A. Bliman. Interval observer for uncertain time-varying SIR-SI model of vector-borne disease. In Proc. of European Control Conference, 2018. URL https://hal.inria.fr/hal-01493078.

S. Audoly, L. D'Angio, M. P. Saccomani, and C. Cobelli. Global identifiability of linear compartmental models-a computer algebra algorithm. IEEE Transactions on Biomedical Engineering, 45(1):36-47, 1998.

N. Bailey. The Mathematical Theory of Infectious Diseases and its Applications. Griffin,London, 1975. 
N. G. Becker. Analysis of infectious disease data, volume 33. CRC Press, 1989.

G. Besançon, editor. Nonlinear observers and applications. Papers based on the presentations at the 28th Grenoble international summer school on control, Grenoble, France, September 200\%. Lecture Notes in Control and Information Sciences 363. Berlin: Springer. xiii, 224 p. , 2007.

D. Bichara, N. Cozic, and A. Iggidr. On the estimation of sequestered infected erythrocytes in Plasmodium falciparum malaria patients. Math. Biosci. Eng., 11(4):741-759, 2014. ISSN 1547-1063. doi: 10.3934/mbe.2014.11.741.

O. N. Bjørnstad, B. F. Finkenstädt, and B. T. Grenfell. Dynamics of measles epidemics: estimating scaling of transmission rates using a time series sir model. Ecological Monographs, 72(2):169-184, 2002.

P.-A. Bliman and B. D'Avila Barros. Interval observers for SIR epidemic models subject to uncertain seasonality. In Positive systems, volume 471 of Lect. Notes Control Inf. Sci., pages 31-39. Springer, Cham, 2017.

P.-A. Bliman, D. Efimov, and R. Ushirobira. A class of nonlinear adaptive observers for sir epidemic model. In Proc. of European Control Conference, 2018.

V. Capasso and V. Capasso. Mathematical structures of epidemic systems, volume 88. Springer, 1993.

S. Cauchemez and N. M. Ferguson. Likelihood-based estimation of continuous-time epidemic models from time-series data: application to measles transmission in london. Journal of the Royal Society Interface, 5(25):885-897, 2008.

S. Cauchemez, F. Carrat, C. Viboud, A. Valleron, and P. Boelle. A bayesian mcmc approach to study transmission of influenza: application to household longitudinal data. Statistics in medicine, 23(22):3469-3487, 2004.

W. Coppel. Dichotomies in Stability Theory, volume 629 of Lecture Notes in Mathematics. Springer-Verlag, Berlin, 1978.

M. De la Sen, A. Ibeas, and S. Alonso-Quesada. Observer-based vaccination strategy for a true mass action SEIR epidemic model with potential estimation of all the populations. Discrete Dyn. Nat. Soc., pages Art. ID 743067, 19, 2011.

M. Diaby, A. Iggidr, and M. Sy. Observer design for a schistosomiasis model. Mathematical Biosciences, 269:17 - 29, 2015. ISSN 0025-5564. doi: http://dx.doi.org/10. 1016/j.mbs.2015.08.008. URL http://www.sciencedirect.com/science/article/ pii/S0025556415001625.

O. Diekmann and J. Heesterbeek. Mathematical epidemiology of infectious diseases: model building, analysis and interpretation. Wiley series in mathematical and computational biology. Wiley, Chichester, 2000. ISBN 0-471-98682-8.

K. Dietz. Transmission and control of arbovirus diseases. In D. Ludwig and K. L. Cooke, editors, Epidemiology, pages 104-121. SIAM, 1975. 
C. Drakeley, P. Corran, P. Coleman, J. Tongren, S. McDonald, I. Carneiro, R. Malima, J. Lusingu, A. Manjurano, W. Nkya, et al. Estimating medium-and long-term trends in malaria transmission by using serological markers of malaria exposure. Proceedings of the National Academy of Sciences of the United States of America, 102(14):51085113, 2005.

L. Esteva and C. Vargas. Analysis of a dengue disease transmission model. Math. Biosci., 150(2):131-151, 1998.

B. F. Finkenstädt and B. T. Grenfell. Time series modelling of childhood diseases: a dynamical systems approach. Journal of the Royal Statistical Society: Series C (Applied Statistics), 49(2):187-205, 2000.

D. Fisman, E. Khoo, and A. Tuite. Early epidemic dynamics of the west african 2014 ebola outbreak: estimates derived with a simple two-parameter model. PLOS currents outbreaks, 2014.

J. Gauthier, H. Hammouri, and S. Othman. A simple observer for nonlinear systems applications to bioreactors. IEEE Trans. Autom. Control, 37(6):875-880, 1992.

P. Gérardin, V. Guernier, J. Perrau, A. Fianu, K. Le Roux, P. Grivard, A. Michault, X. De Lamballerie, A. Flahault, and F. Favier. Estimating chikungunya prevalence in la reunion island outbreak by serosurveys: two methods for two critical times of the epidemic. BMC infectious diseases, 8(1):1, 2008.

A. Guiro, A. Iggidr, D. Ngom, and H. Touré. On the stock estimation for some fishery systems. Rev. Fish Biology and Fisheries, 19(3):313-327, 2009.

H. Guo, M. Y. Li, and Z. Shuai. Global stability of the endemic equilibrium of multigroup SIR epidemic models. Can. Appl. Math. Q, 14(3):259-284, 2006.

H. Guo, M. Y. Li, and Z. Shuai. A Graph-Theoretic Approach to the Method of Global Lyapunov Functions. Proceedings of the American Mathematical Society, 136(8): 2793-2802, 08 2008. doi: 10.2307/20535481. URL http://www.jstor.org/stable/ 20535481.

H. W. Hethcote. The mathematics of infectious diseases. SIAM review, 42(4):599-653, 2000 .

A. Ibeas, M. de la Sen, S. Alonso-Quesada, and I. Zamani. Stability analysis and observer design for discrete-time SEIR epidemic models. Adv. Difference Equ., pages 2015:122, 21,2015 .

A. Iggidr. Encyclopedia of Life Support Systems (EOLSS), chapter Controllability, Observability and Stability of Mathematical Models. UNESCO, Eolss Publishers, Oxford, UK, 2004.

A. Iggidr, G. Sallet, and B. Tsanou. Global stability analysis of a metapopulation SIS epidemic model. Math. Popul. Stud., 19(3):115-129, 2012. http://dx.doi.org/10. 1080/08898480.2012.693844. 
A. Iggidr, G. Sallet, and M. O. Souza. On the dynamics of a class of multi-group models for vector-borne diseases. Journal of Mathematical Analysis and Applications, 441(2):723-743, 2016. ISSN 0022-247X. doi: http://dx.doi.org/10.1016/j. jmaa.2016.04.003. URL http://www.sciencedirect.com/science/article/pii/ S0022247X16300324.

H. Inaba. Threshold and stability results for an age-structured epidemic model. Journal of mathematical biology, 28(4):411-434, 1990.

J. A. Jacquez and P. Greif. Numerical parameter identifiability and estimability: Integrating identifiability, estimability, and optimal sampling design. Mathematical Biosciences, 77(1):201-227, 1985.

J. A. Jacquez et al. Compartmental analysis in biology and medicine. JSTOR, 1985.

L. Joseph, T. W. Gyorkos, and L. Coupal. Bayesian estimation of disease prevalence and the parameters of diagnostic tests in the absence of a gold standard. American Journal of Epidemiology, 141(3):263-272, 1995.

B. Kågström. Bounds and perturbation bounds for the matrix exponential. BIT Numerical Mathematics, 17(1):39-57, 1977. doi: 10.1007/BF01932398. URL http: //dx.doi.org/10.1007/BF01932398.

M. J. Keeling and P. Rohani. Modeling infectious diseases in humans and animals. Princeton University Press, 2008.

W. O. Kermack and A. G. McKendrick. A contribution to the mathematical theory of epidemics. In Proceedings of the Royal Society of London A: mathematical, physical and engineering sciences, volume 115, pages 700-721. The Royal Society, 1927.

T. Kuniya. Global stability analysis with a discretization approach for an age-structured multigroup SIR epidemic model. Nonlinear Analysis: Real World Applications, 12(5): 2640-2655, 2011.

A. Lajmanovich and J. Yorke. A deterministic model for gonorrhea in a nonhomogeneous population. Math. Biosci., 28:221-236, 1976.

A. L. Lloyd. The dependence of viral parameter estimates on the assumed viral life cycle: limitations of studies of viral load data. Proceedings of the Royal Society of London B: Biological Sciences, 268(1469):847-854, 2001.

D. G. Luenberger. An introduction to observers. IEEE Transactions on Automatic Control, AC-16(6):596-602, 1971. URL http://search.ebscohost.com/login.aspx? direct=true\&db=inh\&AN=334765\&amp; lang=fr\&site=ehost - live.

H. Nishiura. Mathematical and statistical analyses of the spread of dengue. Dengue Bulletin, 30:51-67, 2006.

P. Pongsumpun and I. Tang. Transmission of Dengue hemorrhagic fever in an age structured population. Math. Comput. Modelling, 37(9-10):949-961, 2003. 
R. C. Reiner, T. A. Perkins, C. M. Barker, T. Niu, L. F. Chaves, A. M. Ellis, D. B. George, A. Le Menach, J. R. C. Pulliam, D. Bisanzio, C. Buckee, C. Chiyaka, D. A. T. Cummings, A. J. Garcia, M. L. Gatton, P. W. Gething, D. M. Hartley, G. Johnston, E. Y. Klein, E. Michael, S. W. Lindsay, A. L. Lloyd, D. M. Pigott, W. K. Reisen, N. Ruktanonchai, B. K. Singh, A. J. Tatem, U. Kitron, S. I. Hay, T. W. Scott, and D. L. Smith. A systematic review of mathematical models of mosquito-borne pathogen transmission: 1970-2010. Journal of The Royal Society Interface, 10(81), 2013. doi: 10.1098/rsif.2012.0921.

F. Rocha, M. Aguiar, M. O. Souza, and N. Stollenwerk. Time-scale separation and center manifold analysis describing vector-borne disease dynamics. International Journal of Computer Mathematics, 90(10):2105-2125, 2013.

R. Ross. The prevention of malaria. John Murray, 1911.

D. L. Smith, K. E. Battle, S. I. Hay, C. M. Barker, T. W. Scott, and F. E. McKenzie. Ross, macdonald, and a theory for the dynamics and control of mosquito-transmitted pathogens. PLoS Pathog, 8(4):e1002588 EP -, 04 2012. URL http://dx.doi.org/ 10.1371\%2F journal.ppat. 1002588 .

H. L. Smith. Monotone dynamical systems: an introduction to the theory of competitive and cooperative systems. Mathematical Surveys and Monographs. 41. Providence, RI: American Mathematical Society (AMS). x, 174 p. , 1995.

M. O. Souza. Multiscale analysis for a vector-borne epidemic model. Journal of Mathematical Biology, 68(5):1269-1293, 2014.

R. Tami, D. Boutat, and G. Zheng. Extended output depending normal form. Automatica, 49(7):2192-2198, 2013. doi: http://dx.doi.org/10.1016/j. automatica.2013.03.025. URL http://www.sciencedirect.com/science/article/ $\mathrm{pii/S0005109813001945.}$

R. Tami, D. Boutat, G. Zheng, and F. Kratz. Parameters and states estimation for dengue epidemic model. In 2014 European Control Conference (ECC), pages 528533, June 2014. doi: 10.1109/ECC.2014.6862173.

T. Toni, D. Welch, N. Strelkowa, A. Ipsen, and M. P. Stumpf. Approximate bayesian computation scheme for parameter inference and model selection in dynamical systems. Journal of the Royal Society Interface, 6(31):187-202, 2009.

S. Towers, O. Patterson-Lomba, and C. Castillo-Chavez. Temporal variations in the effective reproduction number of the 2014 west africa ebola outbreak. PLOS Currents Outbreaks, 2014.

J. X. Velasco-Hernández, J. A. García, and D. Kirschner. Remarks on modeling hostviral dynamics and treatment. In C. e. e. a. Castillo-Chavez, editor, Mathematical approaches for emerging and reemerging infectious diseases: An introduction. Proceedings of a tutorial Introduction to epidemiology and immunology, pages 287-308. Springer. IMA Vol. Math. Appl. 125, 2002.

W. Wonham. Linear multivariable control: a geometric approach. 2nd ed. , 1979. 
Appendix A. A glimpse at the DAta 
Date (month) 1 Number of new cases Proportion of new case

\begin{tabular}{|c|c|c|}
\hline & & \\
\hline 0 & 129 & 0.0000222 \\
\hline 1 & 127 & 0.0000219 \\
\hline 2 & 273 & 0.0000471 \\
\hline 3 & 501 & 0.0000864 \\
\hline 4 & 683 & 0.0001178 \\
\hline 5 & 265 & 0.0000457 \\
\hline 6 & 88 & 0.0000152 \\
\hline 7 & 31 & 0.0000053 \\
\hline 8 & 26 & 0.0000045 \\
\hline 9 & 25 & 0.0000043 \\
\hline 10 & 24 & 0.0000041 \\
\hline 11 & 26 & 0.0000045 \\
\hline 12 & 500 & 0.0000862 \\
\hline 13 & 1144 & 0.0001972 \\
\hline 14 & 2234 & 0.0003852 \\
\hline 15 & 6731 & 0.0011605 \\
\hline 16 & 7364 & 0.0012697 \\
\hline 17 & 3613 & 0.0006229 \\
\hline 18 & 1115 & 0.0001922 \\
\hline 19 & 430 & 0.0000741 \\
\hline 20 & 278 & 0.0000479 \\
\hline 21 & 398 & 0.0000686 \\
\hline 22 & 497 & 0.0000857 \\
\hline 23 & 1552 & 0.0002676 \\
\hline 24 & 25966 & 0.0044769 \\
\hline 25 & 41101 & 0.0070864 \\
\hline 26 & 52032 & 0.0089710 \\
\hline 27 & 9942 & 0.0017141 \\
\hline 28 & 1368 & 0.0002359 \\
\hline 29 & 195 & 0.0000336 \\
\hline 30 & 87 & 0.0000150 \\
\hline$\vdots$ & $\vdots$ & $\vdots$ \\
\hline 92 & 461 & 0.0000795 \\
\hline 93 & 672 & 0.0001159 \\
\hline 94 & 1519 & 0.0002619 \\
\hline 95 & 2307 & 0.0003978 \\
\hline 96 & 11445 & 0.0019733 \\
\hline 97 & 15131 & 0.0026088 \\
\hline 98 & 40485 & 0.0069802 \\
\hline 99 & 32534 & 0.0056093 \\
\hline 100 & 4619 & 0.0007964 \\
\hline 101 & 401 & 0.0000691 \\
\hline
\end{tabular}

TABLE 1. Number of reported in the district of Jacarepaguá, together with the corresponding proportion - the population of this district was taken from the Brazilian census of 2000 as 540000 . 